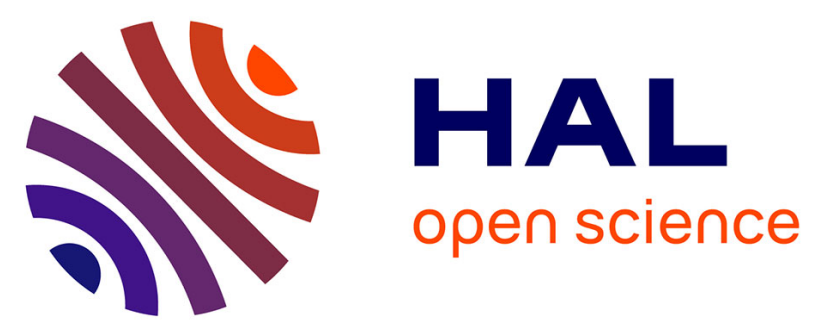

\title{
2,4-Bis(fluoroalkyl)quinoline-3-carboxylates as Tools for the Development of Potential Agrochemical Ingredients
}

Fallia Aribi, Armen Panossian, Jean-Pierre Vors, Sergiy Pazenok, Frédéric R. Leroux

\section{- To cite this version:}

Fallia Aribi, Armen Panossian, Jean-Pierre Vors, Sergiy Pazenok, Frédéric R. Leroux. 2,4Bis(fluoroalkyl)quinoline-3-carboxylates as Tools for the Development of Potential Agrochemical Ingredients. European Journal of Organic Chemistry, 2018, Organofluorine Chemistry in Europe, 2018 (27-28), pp.3792-3802. 10.1002/ejoc.201800375 . hal-02105501

\section{HAL Id: hal-02105501 \\ https://hal.science/hal-02105501}

Submitted on 13 Nov 2020

HAL is a multi-disciplinary open access archive for the deposit and dissemination of scientific research documents, whether they are published or not. The documents may come from teaching and research institutions in France or abroad, or from public or private research centers.
L'archive ouverte pluridisciplinaire HAL, est destinée au dépôt et à la diffusion de documents scientifiques de niveau recherche, publiés ou non, émanant des établissements d'enseignement et de recherche français ou étrangers, des laboratoires publics ou privés. 


\title{
2,4-Bis(fluoroalkyl)quinoline-3-carboxylates as tools for the development of potential agrochemical ingredients
}

\author{
Fallia Aribi, ${ }^{[a, b]}$ Armen Panossian, ${ }^{[a, b]}$ Jean-Pierre Vors, ${ }^{[b, c]}$ Sergii Pazenok, ${ }^{[b, d]}$ and Frédéric R. \\ Leroux $^{\star[a, b]}$
}

\begin{abstract}
From an easy and scalable synthetic access to quinoline derivatives substituted by fluorinated groups in both C2- and C4positions developed in our laboratory, we devised the synthesis of a new series of unprecedented 2,4-bis(fluoroalkyl)quinoline-3carboxylates in two steps only. After standard saponification, the latter afforded their corresponding 2,4-bis(fluoroalkyl)quinoline-3-carboxylic acids, which served as pivotal intermediates for post-functionalization reactions. Indeed, the carboxylic function could then be derived according to known procedures and allowed the introduction of chemical diversity in C3-position of these unprecedented structures. The resulting highly functionalized quinolines will then serve as platform in the development of ingredients with strong potential for agrochemical research.
\end{abstract}

\section{Introduction}

Introduction of fluoroalkyl groups into organic molecules in a selective and efficient way has piqued the interest of chemists, not only because of its synthetic challenge, but also because fluoro-organic compounds, when compared to their nonfluorinated analogues, often show unique biological properties useful in medicinal and agrochemical chemistry, as well as in materials science. ${ }^{[1]}$ Indeed, because of the strong electronic perturbation induced by fluorine atoms and fluorinated groups, organic molecules are subjected to drastic changes of their physico-chemical properties. For that reason, general methodologies for the synthesis of fluoroalkylated compounds are highly demanded. ${ }^{[2]}$

Recently, bis-fluorinated quinolines have been increasingly investigated in literature. ${ }^{[2 f, 3]}$ This sudden interest for such compounds is caused by the fact that, on the one hand, the quinoline moiety is frequently found among active ingredients, especially therapeutic drugs, ${ }^{[4]}$ and, on the other hand, fluoroalkyl groups appended to heteroaromatics may enhance the lipophilicity and oxidative stability of bioactive molecules. ${ }^{[5]}$ Despite this interest, only few series of quinolines substituted in C2- and C4-positions by fluorinated groups have been reported, ${ }^{[3 \mathrm{c} \text {, }}$ ${ }^{3 e, 6]}$ and even fewer of them bear substituents in C3-position. ${ }^{[3 a, 7]}$ However, it seems that such substitution in C3-position of quinolines already stands out as potentially attractive. ${ }^{[3 a]}$

We had already reported on the easy and modular access to 2,4bis(fluoroalkylated) quinoline derivatives with various substitution on the benzo ring. ${ }^{[6]}$ Their synthesis (Scheme 1) relied on 1) the condensation of $N$-aryl $\alpha$-fluoroacetimines onto highly reactive acyl fluoride equivalents, namely 1-fluoro-1-(fluoroalkyl)- $N, N$ dialkyliminium salts obtained by treatment of FluoroalkylAmino Reagents (FARs, Figure 1) by a Lewis acid; and 2) on the subsequent cyclization of the resulting vinamidinium in a Combes-like reaction. In fact, apart from quinolines, FARs allow the access to various types of new fluorinated heterocycles, as we had also demonstrated. ${ }^{[6,8]}$ Three of these reagents are commercially available: 1,1,2,2-tetrafluoro- $N, N$-dimethylethan-1amine (1A; TFEDMA, Petrov reagent), ${ }^{[9]}$ 2-chloro- $\mathrm{N}, \mathrm{N}$-diethyl1,1,2-trifluoroethan-1-amine (1B; Yarovenko reagent), ${ }^{[10]}$ and $\mathrm{N}, \mathrm{N}$-diethyl-1,1,2,3,3,3-hexafluoropropan-1-amine (1C; Ishikawa reagent). ${ }^{[11]}$ Additionally, we developed a new FAR 1D, which showed similar reactivity to TFEDMA $1 \mathrm{~A}$ and allowed the introduction of the $-\mathrm{CHFOCF}_{3}$ group (Figure 1). ${ }^{[8 \mathrm{~d}, 8 \mathrm{8f}]}$

Herein, we wish to describe the synthesis of new 2,4bis(fluoroalkyl)quinolines substituted in the aforementioned interesting C3-position, with an effort to introduce chemical diversity. Indeed, the purpose was to design molecules which will serve as platform for further functionalization towards active agrochemical ingredients.

[a] Dr. F. Aribi, Dr. A. Panossian, Dr. F. R. Leroux

Université de Strasbourg, Université de Haute-Alsace, CNRS

LIMA UMR 7042, F-67000 Strasbourg, France

E-mail: frederic.leroux@unistra.fr; lima.unistra.fr

[b] Dr. F. Aribi, Dr. A. Panossian, Dr. F. Leroux, Dr. J.-P. Vors, Dr. S.

Pazenok

Joint laboratory Unistra-CNRS-Bayer (Chemistry of Organofluorine

Compounds), France.

[c] Dr. J.-P. Vors

Bayer S.A.S.

14 Impasse Pierre Baizet, BP99163, 69263 Lyon Cedex 09, France.

[d] Dr. S. Pazenok

Bayer AG

Alfred-Nobel-Strasse 50, 40789 Monheim, Germany

Supporting information for this article is given via a link at the end of the document. 


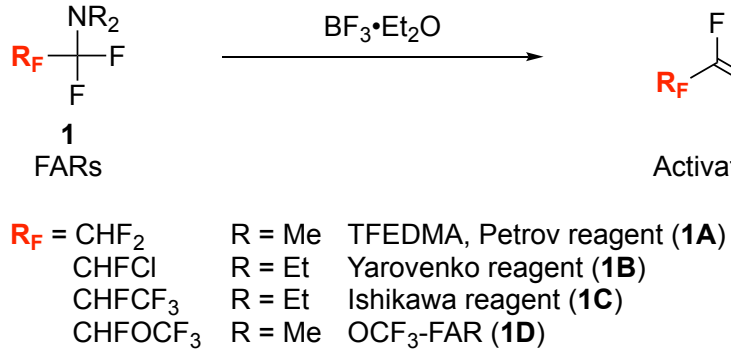

Figure 1: Fluoroalkyl Amino Reagents (FARs)

\section{Results and Discussion}

To access the desired quinolines with diverse functionalization in C3-position, we first performed the synthesis of the corresponding quinoline-3-carboxylates 5 . Towards this aim, we followed a strategy similar to the previous one, but starting from fluorinated ethyl acetoacetates (Scheme 1). Their condensation with aniline derivatives provided the expected enamines 3 (Scheme 2).

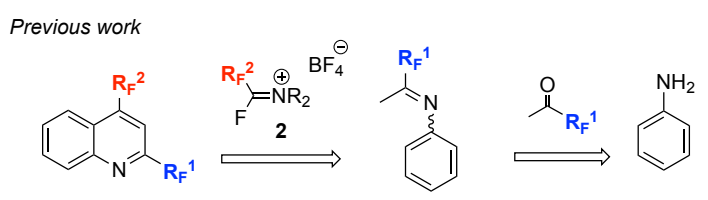

$$
\text { (1) }
$$

Scheme 1: Retrosynthetic scheme of 2,4-bis(fluoroalkyl)-3-substituted quinoline derivatives

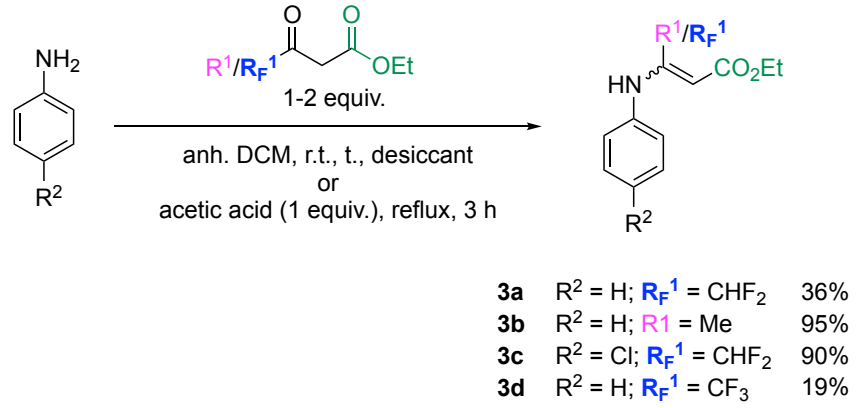

Scheme 2: Synthesis of fluorinated enamines 3

Enamines $\mathbf{3 b}$ and $\mathbf{3 c}$ were obtained in almost quantitative yields. These molecules appeared sensitive to moisture and silica, undergoing degradation in certain cases accompanied by the recovery of unknown fluorinated species; consequently, they were used without being further purified. The synthesis of 4,4difluoro- $N$-phenylaminobutanoate $\mathbf{3 a}$ was carried out according to the conditions developed by Perrone et al. ${ }^{[12]}$ A mixture of the desired compound and unidentified fluorinated species was recovered even after purification by distillation of the starting fluoroacetoacetate. We also attempted to purify the reactional mixture by column chromatography; however, even though compound 3a was collected pure, a non-negligible amount of degraded product was also recovered, which considerably decreased the yield of the reaction. Following the same procedure, 3d was obtained in only negligible amount. The starting aniline, ethyl $\alpha^{\prime}, \alpha^{\prime}, \alpha^{\prime}$-trifluoroacetoacetate and degradation products were often recovered. To account for these results, we assume that the latter trifluoroacetoacetate predominantly exists under its enol form, which can impede aniline condensation. On the other hand, we could obtain compound $\mathbf{3} \mathbf{d}$ in refluxing acetic acid according to the procedure of Sharada et al. ${ }^{[13]}$ However, in our hands, only $19 \%$ yield of product was obtained compared to the $63 \%$ of literature. Analysis of $\mathbf{3 d}$ by ${ }^{1} \mathrm{H}-\mathrm{NMR}$ showed an imine-enamine equilibrium, which rapidly shifted towards the enamine form only.

The second step was the nucleophilic attack of activated FARs 2 by enamines 3 to afford quinoline derivatives 5 (Scheme 3). Good yields, ranging from 74 to $97 \%$ were obtained for compounds $5 \mathrm{aA}$, $\mathbf{5 d A}, \mathbf{5 a D}, \mathbf{5 d D}$. These molecules bear either a $-\mathrm{CHF}_{2}$ or a $\mathrm{CHFOCF}_{3}$ moiety in C4-position and they were obtained after in situ cyclization of the vinamidinium intermediate $\mathbf{4}$ and purification by column chromatography. 


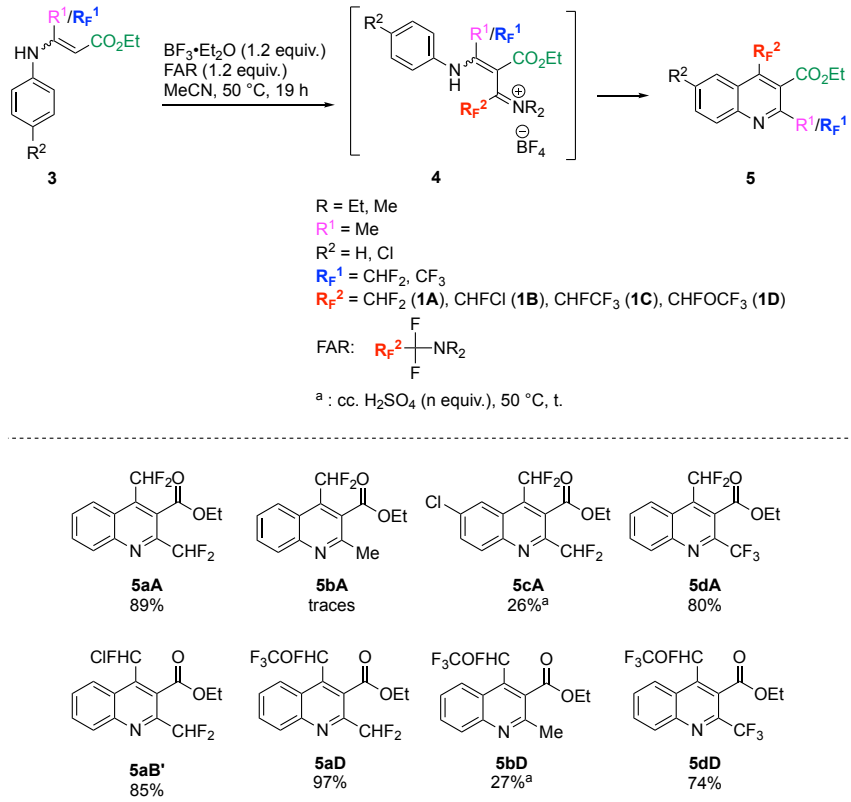

Scheme 3: Synthesis of quinoline derivatives 5 bearing a carboxylate group in position 3

Conversely, according to GCMS and ${ }^{1} \mathrm{H}$-NMR analysis, the attempted synthesis of 5cA led to a mixture of acetamide $14-$ which results from the reaction of activated TFEDMA 2A with $p$ chloroaniline, released by the cleavage of enamine $3 c-$ and vinamide $15 \mathrm{cA}$-which corresponds to the hydrolysed vinamidinium intermediate-, with only traces of the targeted quinoline. After acidic treatment with 10 equiv. of concentrated sulfuric acid, we difficultly managed to reach $26 \%$ yield of the desired product. Indeed, despite the excess of acid, a substantial amount of 14 and 15cA was still recovered (Scheme 4).

$$
\text { (2) }
$$

Scheme 4: Synthesis of ethyl 6-chloro-2,4-bis(difluoromethyl)quinoline-3carboxylate 5cA

On the other hand, the synthesis of $\mathbf{5 b A}$, starting from a nonfluorinated acetoacetate remained unsuccessful despite the addition of 20 equiv. of concentrated $\mathrm{H}_{2} \mathrm{SO}_{4}$ in the reaction mixture. Although we observed by ${ }^{1} \mathrm{H}-\mathrm{NMR}$ the formation of the vinamide intermediate $15 \mathrm{bA}$, the addition of acid did not alter sensibly the unsuccessful outcome of the cyclization process
(Scheme 5). This result contrasts dramatically with the good yield $(89 \%)$ of the closely related compound $\mathbf{5 a A}$, bearing a second difluoromethyl group instead of the methyl one. A partial explanation would incriminate the inductively electron-donating terminal methyl group, which may decrease the electrophilic character of the distal carbon being attacked by the phenyl ring; however, unlike the $\mathrm{CHF}_{2}$ group, the methyl group should also enrich the aniline nitrogen, hence the nucleophilicity of the arene. Thus, as we had described previously, ${ }^{[6]}$ counter-operating effects of a same substituent makes rationalization of the cyclization of vinamides or vinamidiniums into the desired quinolines quite difficult. Similarly, one can only speculate on the reasons for which quinoline $5 \mathrm{bD}$ could be obtained in $27 \%$ yield, by using activated $\mathrm{OCF}_{3}-\mathrm{FAR} 2 \mathrm{D}$, while $5 \mathrm{bA}$ failed to be produced.

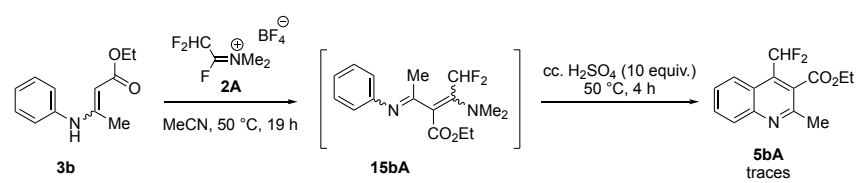

Scheme 5: Attempt to synthesize compound 5bA

Finally, when starting from the Yarovenko reagent 1B, the reaction with $3 \mathbf{a}$ led mostly to the degradation of the latter starting material and to the recovery of the hydrolysed FAR. Suspecting that the presence of less bulky substituents on the nitrogen atom of the FAR could enhance the reactivity of the corresponding iminium, ${ }^{[8]}$ we freshly prepared the $\mathrm{N}, \mathrm{N}$-dimethyl analogue 1B' of Yarovenko's reagent 1B, which, upon reaction with $\mathbf{3 a}$, successfully afforded $\mathbf{5 a B}$ ' in $85 \%$ yield (Scheme $\mathbf{6}$ ).

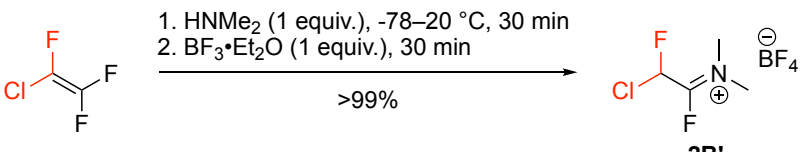

2B'<smiles>C#C/C(=C/C(=O)OCC)Nc1ccccc1</smiles>

3a

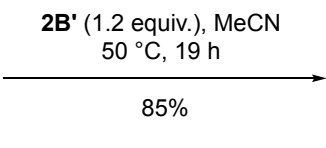<smiles>CCOC(=O)c1c(C(F)F)nc2ccccc2c1C(F)Cl</smiles>

$5 \mathrm{aB}^{\prime}$
Scheme 6: Synthesis of 5aB' from the $N, N$-dimethyl analogue of the activated Yarovenko reagent $\mathbf{B . 2}$

The next part of this work was the functionalization of the synthesized quinolines in C3-position. First, we saponified selected compounds 5aA, 5aD and 5cA using potassium hydroxide in ethanol and water $(60: 40)$ at $80^{\circ} \mathrm{C}$ (Scheme 7). The reaction afforded carboxylic acids $6 \mathrm{aA}$ and $\mathbf{6 a D}$ efficiently, while $6 \mathrm{cA}$ was obtained in moderate yield only. 
<smiles>[R]c1ccc2nc(C(F)F)c(C(=O)[O-])c([R7])c2c1</smiles>

5<smiles>O=C(O)c1c(C(F)F)nc2ccccc2c1C(F)F</smiles>

$6 \mathrm{aA}$ $6 \mathrm{aA}$
$99 \%$
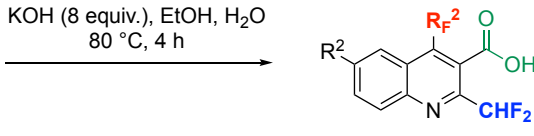<smiles>O=C(O)c1c(C(F)F)nc2ccc(Cl)cc2c1C(F)F</smiles>

Scheme 7: Saponification of selected esters

Second, we investigated on the conversion of the carboxylic acid function into other functional groups. 2,4Bis(difluoromethyl)quinoline-3-carboxylic acid $\mathbf{6 a A}$ was used as a model compound to undergo a Curtius rearrangement in presence of diphenylphosphoryl azide (DPPA). The resulting isocyanate intermediate was trapped by $t-\mathrm{BuOH}$ to provide the corresponding $t$-butyl (2,4-bis(difluoromethyl)quinolin-3yl)carbamate $7 \mathrm{aA}$ in $64 \%$ yield. The amine moiety was then deprotected in acidic medium using TFA and 2,4bis(difluoromethyl)quinolin-3-amine $\mathbf{8 a A}$ was obtained in quantitative yield. This 2 -step sequence $(63 \%$ yield) was also performed in one pot following the procedure of Miller et al. ${ }^{[14]}$ in $61 \%$ yield (Scheme 8).

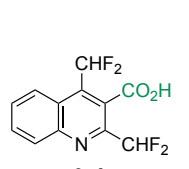

6aA
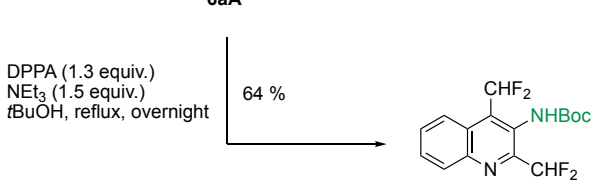

$7 \mathrm{aA}$

Scheme 8: Curtius rearrangement of compound $6 \mathbf{a A}$

Having installed an amino group in C3-position, we envisaged its transformation to several other groups by means of Sandmeyer reactions. In a first attempt, following the procedure of Bui et al. ${ }^{[15]}$ using sodium nitrite, a mixture of copper bromide and hydrobromic acid, only the starting material was recovered. When using $t$-butyl nitrite instead, following the work of Boezio et al., ${ }^{[16]}$ and several copper sources, we accessed 2,4bis(difluoromethyl)-3-iodoquinoline $\quad 9 a A, \quad 3$-bromo-2,4bis(difluoromethyl)quinoline $10 \mathrm{aA}$ and 2,4bis(difluoromethyl)quinoline-3-carbonitrile $11 \mathrm{aA}$ with moderate yields (Scheme $\mathbf{9}$ ).<smiles>Nc1c(C(F)F)nc2ccccc2c1C(F)F</smiles>

$8 \mathrm{aA}$
CuY or $\mathrm{CuX}_{\mathrm{n}}$ (n equiv.)

$t$-BuONO (4 equiv.)

$\mathrm{MeCN}$

r.t. to $60^{\circ} \mathrm{C}, \mathrm{t}$

$\mathrm{CuX}_{\mathrm{n}}=\mathrm{Cul}, \mathrm{CuBr}_{2}$

$\mathrm{CuY}=\mathrm{CuCN}$<smiles>[Y10]c1c(CF)nc2ccccc2c1CF</smiles>

$X=1 \quad 9 a A(65 \%)$

$X=B r \quad 10 \mathrm{aA}(50 \%)$

$\mathrm{Y}=\mathrm{CN} \quad 11 \mathrm{aA}(47 \%)$
Scheme 9: Sandmeyer reactions of aminoquinoline $8 \mathbf{a A}$

Last but not least, we used in turn the iodo derivative 9aA to access the corresponding pinacolboronic ester. Thus, 9aA underwent halogen/metal exchange using $\mathrm{iPrMgCl} \cdot \mathrm{LiCl}$ and in situ trapping with isopropoxypinacolborane following the procedure of Chavant et al. ${ }^{[17]}$ 2,4-Bis(difluoromethyl)-3(pinacolboranyl)quinoline 12aA was obtained in $54 \%$ yield, along with the hydrolysed compound $13 \mathrm{aA}$, which we had already described, ${ }^{[6]}$ as sole remnant of the starting material (Scheme 10).

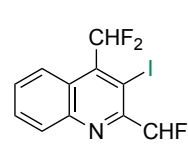

$9 \mathrm{aA}$

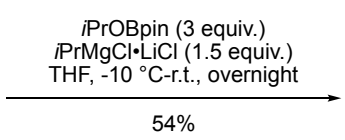

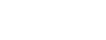<smiles>Cc1c(B2OC(C)(C)C(C)(C)O2)c(C(F)F)nc2ccccc12</smiles>

$12 \mathrm{aA}$<smiles>FC(F)c1cc(C(F)F)c2ccccc2n1</smiles>

Scheme 10: Halogen/metal interconversion-borylation sequence

Thus, thanks to model substrate $\mathbf{5 a A}$, we showed that we could access several key 2,4-bis(fluoroalkyl)quinoline building blocks, with diverse substitution in C3-position by synthetically useful functional groups. These compounds could be obtained from FARs, anilines and fluorinated acetoacetates in two to seven steps, with an overall yield comprised between 7 and $13 \%$, by means of generally easy-to-implement reactions.

\section{Conclusions}

We developed an access to a series of unprecedented ethyl 2,4bis(fluoroalkyl)quinoline-3-carboxylates, whose fluoroalkyl groups are either identical or not, in two steps starting from available or easily accessible reagents. Most carboxylates were formed in good yields. Interestingly, when the intermediate $N$-aryl- $\beta$ aminoacrylates were non-fluorinated, the expected quinoline-3carboxylates bearing only one fluoroalkyl group in C4-position could not be obtained. We also demonstrated that, using known methodologies, the resulting ethyl quinoline-3-carboxylates can serve as platform towards various highly useful 2,4bis(fluoroalkyl)quinoline building blocks, bearing versatile 
carboxylic acid, amino, halogeno, cyano or pinacolboranyl groups in C3-position. These key compounds open avenues to further derivation. Finally, it would be of great interest to develop new FARs, as already attempted by Maslennikov et al., ${ }^{[18]}$ in order to access quinoline derivatives bearing other fluorinated moieties in C4-position.

\section{Experimental Section}

General remarks: All reactions were performed in flame-dried glassware using Schlenk techniques for reactions needing anhydrous conditions. Liquids and solutions were transferred with syringes. Air- and moisture- sensitive materials were stored and handled under an atmosphere of argon. Solvents were purified and dried following standard procedures: Dichloromethane (DCM) and Tetrahydrofuran (THF) were respectively distilled from $\mathrm{CaH}_{2}$ or sodium + benzophenone prior to use. Desiccants (4 $\AA$ molecular sieves (4 $\AA$ MS) were previously activated in an oven. Technical grade solvents for extraction and chromatography (cyclohexane, dichloromethane, $n$-pentane, ether, toluene, and ethyl acetate) were used without purification. Starting materials, if commercial, were purchased from standard suppliers (SigmaAldrich, Acros, Alfa Aesar and Apollo scientific) and used as such, provided that adequate checks (NMR) had confirmed the claimed purity. Analytical thin-layer chromatography (TLC) was carried out on $0.25 \mathrm{~mm}$ Merck silica-gel (60-F254). Flash column chromatography was performed on silica gel $60(40-63 \mu \mathrm{m}, 230$ 400 mesh, ASTM) by Merck using the indicated solvents. ${ }^{1} \mathrm{H},{ }^{13} \mathrm{C}$, and ${ }^{19} \mathrm{~F}-\mathrm{NMR}$ spectra were recorded in $\mathrm{CDCl}_{3}$, acetone- $\mathrm{d}_{6}$ and DMSO-d 6 on Bruker AV 400 instruments $\left({ }^{1} \mathrm{H}: 400 \mathrm{MHz},{ }^{19} \mathrm{~F}: 376\right.$ $\mathrm{MHz},{ }^{13} \mathrm{C}: 101 \mathrm{MHz}$ ). Chemical shifts are reported in parts per million (ppm) and are referenced to the residual solvent resonance as the internal standard (chloroform $\left(\delta\left[{ }^{1} \mathrm{H}\right]=7.26\right.$ and accordingly $\delta\left[{ }^{13} \mathrm{C}\right]=77.16 \mathrm{ppm}$ ). Data are reported as follows: chemical shift, multiplicity ( $\mathrm{br} \mathrm{s}=$ broad singlet, $\mathrm{s}=$ singlet, $\mathrm{d}=$ doublet, $\mathrm{t}=$ triplet, $\mathrm{q}=$ quartet, $\mathrm{m}=$ multiplet, $\mathrm{qd}=$ quadruplet of doublets, $\mathrm{td}=$ triplet of doublets, $\mathrm{dd}=$ doublet of doublets, $\mathrm{dq}=$ doublet of quadruplets), coupling constant $(\mathrm{Hz})$ and integration. Spectra were processed with the program MestReNova (Version 6.0.2-5475). Melting points (MP) were determined for crystalline compounds with a Büchi Melting Point Apparatus M-560 and are not corrected. IR spectra were measured with a Perkin Elmer Spectrum UATR two (diamond detection). HRMS analysis (measurement accuracy $\leq 15 \mathrm{ppm}$ ) and EA were performed by the analytical facility at the University of Strasbourg. Crystal X-ray diffraction analysis was carried out by the Radiocrystallography Service of the University of Strasbourg.

\section{SYNTHESIS OF FLUOROALKYL AMINO REAGENT-DERIVED FLUOROIMINIUM SALTS}

$N$-(2-Chloro-1,2-difluoroethylidene)- $N$ methylmethanaminium tetrafluoroborate 2B'

1-Chloro-1,2,2-trifluoroethene (1 equiv., $0.195 \mathrm{~mL}, 2.58 \mathrm{mmol}$ ) was liquefied in a Schlenk apparatus under argon at $-78{ }^{\circ} \mathrm{C}$.
Dimethylamine (1 equiv., $2 \mathrm{M}$ in THF, $1.29 \mathrm{~mL}, 2.58 \mathrm{mmol}$ ) was added slowly via syringe at $-78^{\circ} \mathrm{C}$. After $5 \mathrm{~min}$, the cold bath was replaced by a water bath and the mixture was stirred for $15 \mathrm{~min}$. Boron trifluoride diethyl etherate $\left(\mathrm{BF}_{3}{ }^{\bullet} \mathrm{Et}_{2} \mathrm{O}\right)$ ( 1 equiv., $0.328 \mathrm{~mL}$, $2.59 \mathrm{mmol}$ ) was added via syringe and the reaction mixture was stirred for $30 \mathrm{~min}$. Anhydrous DCM was then added to precipitate the desired salts, and the supernatant was removed; in case of polymerization, addition of anhydrous acetonitrile solubilized the mixture which was then used as such. Yield of 2B' was estimated to be of $99 \%$ according to ${ }^{1} \mathrm{H}$ NMR analysis. ${ }^{1} \mathrm{H}$ NMR $(400 \mathrm{MHz}$, DMSO) $\delta_{\mathrm{H}}=7.16\left(\mathrm{~d},{ }^{2} \mathrm{~J}_{\mathrm{H}-\mathrm{F}}=48.8 \mathrm{~Hz}, 1 \mathrm{H}, \mathrm{CHFCl}\right), 2.97$ and 2.87 $\left(2{ }^{*} \mathrm{~s}, 6 \mathrm{H}, \mathrm{N}\left(\mathrm{CH}_{3}\right)_{2}{ }^{+}\right)$ppm. ${ }^{19} \mathrm{~F} \mathrm{NMR}(376 \mathrm{MHz}, \mathrm{DMSO}) \delta_{\mathrm{F}}=-$ $144.34\left(\mathrm{~d},{ }^{2} \mathrm{~J}_{\mathrm{F}-\mathrm{H}}=48.8 \mathrm{~Hz}, \mathrm{CHFCl}\right),-148.03$ (s, N=CF), -148.08 (s, $\left.\mathrm{BF}_{4}^{-}\right) \mathrm{ppm}$.

${ }^{13} \mathrm{C}$ NMR analysis was carried out on the corresponding amide after hydrolysis.

${ }^{13} \mathrm{C}$ NMR $(126 \mathrm{MHz}, \mathrm{DMSO}) \delta_{\mathrm{C}}=163.20\left(\mathrm{~d},{ }^{2} \mathrm{~J}_{\mathrm{C}-\mathrm{F}}=23.9 \mathrm{~Hz}, \mathrm{CO}\right)$, $91.52\left(\mathrm{~d},{ }^{1} \mathrm{~J}_{\mathrm{C}-\mathrm{F}}=244.8 \mathrm{~Hz}, \mathrm{CHFCl}\right), 36.26$ and $35.70\left(2{ }^{*} \mathrm{~s}, \mathrm{CH}_{3}\right)$ ppm.

$N$-(1,2-Difluoro-2-(trifluoromethoxy)ethylidene)- $N$ methylmethanaminium tetrafluoroborate 2D

1,1,2-Trifluoro-2-(trifluoromethoxy)ethene (1 equiv., $0.8 \mathrm{~mL}, 7.23$ $\mathrm{mmol}$ ) was liquefied in a Schlenk apparatus under argon at $-78^{\circ} \mathrm{C}$. Dimethylamine (1 equiv., $2 \mathrm{M}$ in THF, $3.62 \mathrm{~mL}, 7.24 \mathrm{mmol}$ ) was added slowly via syringe at $-78{ }^{\circ} \mathrm{C}$. After $5 \mathrm{~min}$, the cold bath was replaced by a water bath and the mixture was stirred for $15 \mathrm{~min}$. $\mathrm{BF}_{3} \cdot \mathrm{Et}_{2} \mathrm{O}$ (1 equiv., $0.92 \mathrm{~mL}, 7.26 \mathrm{mmol}$ ) was added via syringe and the reaction mixture was stirred for $30 \mathrm{~min}$. The yield of the desired $\quad \mathrm{N}$-(1,2-difluoro-2-(trifluoromethoxy)ethylidene)- $\mathrm{N}$ methylmethanaminium tetrafluoroborate (2D) was estimated to be of $85 \%$ by ${ }^{1} \mathrm{H}$ NMR analysis and the compound was used directly in the next step.

\section{SYNTHESIS OF ENAMINES FROM FLUORINATED ACETOACETATES}

Typical procedure A for the synthesis of enamines derivatives (3)

Under argon atmosphere, an excess of cold fluorinated ethyl acetoacetate (1 equiv.) was added to the aniline derivative (1 equiv. ) in anhydrous DCM $(2 \mathrm{~mL} / 1 \mathrm{mmol})$ in presence of desiccant (e.g. $4 \AA \mathrm{MS}$ ). The reaction mixture was stirred for the indicated time at room temperature. The desiccant was then filtered off on a Celite ${ }^{\circledR}$ pad, which was washed with ether. The filtrate was concentrated under reduced pressure to provide the desired product.

The following experiments were carried out according to Typical Procedure $\boldsymbol{A}$, and specific details are reported as: a) commercially available perfluoroacetoacetate; b) aniline derivative; c) desiccant and time; and d) yield and aspect. Individual analysis for each compound $\mathbf{3}$ is given below. Atoms are numbered in the description of NMR spectra according to the Supporting Information.

Ethyl 4,4-difluoro-3-(phenylamino)but-2-enoate 3a 
a) Ethyl 4,4-difluoroacetoacetate (1 equiv., $8.43 \mathrm{~mL}, 64.4 \mathrm{mmol}$ ); b) Aniline (1 equiv., $5.87 \mathrm{~mL}, 64.4 \mathrm{mmol}$ ); c) $4 \AA \mathrm{MS}$, for $4 \mathrm{~h}$; d) Ethyl 4,4-difluoro-3-(phenylamino)but-2-enoate $\mathbf{3 a}$ was obtained in $36 \%$ yield $(5.63 \mathrm{~g})$ as a colourless oil after purification by column chromatography using a gradient of AcOEt in pentane (2$98 \%$ ). Compound $3 \mathrm{a}$ was used directly without any further purification to avoid its degradation on silica gel column. ${ }^{1} \mathrm{H}$ NMR $\left(400 \mathrm{MHz}, \mathrm{CDCl}_{3}\right) \delta_{\mathrm{H}}=9.93(\mathrm{~s}, 1 \mathrm{H}, \mathrm{NH}), 7.36\left(\mathrm{t},{ }^{3} \mathrm{~J}_{\mathrm{H}-\mathrm{H}}=7.8 \mathrm{~Hz}\right.$, $\left.2 \mathrm{H}, \mathrm{C}_{3}, 5 \mathrm{H}\right), 7.22\left(\mathrm{t},{ }^{3} \mathrm{~J}_{\mathrm{H}-\mathrm{H}}=7.4 \mathrm{~Hz}, 1 \mathrm{H}, \mathrm{C}_{4} \mathrm{H}\right), 7.15\left(\mathrm{~d},{ }^{3} \mathrm{~J}_{\mathrm{H}-\mathrm{H}}=7.6\right.$ $\left.\mathrm{Hz}, 2 \mathrm{H}, \mathrm{C}_{2,}{ }_{6} \mathrm{H}\right), 6.21\left(\mathrm{t},{ }^{2} \mathrm{~J}_{\mathrm{H}-\mathrm{F}}=53.3 \mathrm{~Hz}, 1 \mathrm{H}, \mathrm{C}_{7} \mathrm{CHF}_{2}\right), 5.26(\mathrm{~s}, 1 \mathrm{H}$, $\left.\mathrm{C}_{8} \mathrm{H}\right), 4.20\left(\mathrm{q},{ }^{3} \mathrm{~J}_{\mathrm{H}-\mathrm{H}}=7.1 \mathrm{~Hz}, 2 \mathrm{H}, \mathrm{C}_{9} \mathrm{OCH}_{2} \mathrm{CH}_{3}\right), 1.31\left(\mathrm{t},{ }^{3} \mathrm{~J}_{\mathrm{H}-\mathrm{H}}=7.1\right.$ $\left.\mathrm{Hz}, 3 \mathrm{H}, \mathrm{C}_{9} \mathrm{OCH}_{2} \mathrm{CH}_{3}\right)$ ppm. ${ }^{19} \mathrm{~F} \mathrm{NMR}\left(376 \mathrm{MHz}, \mathrm{CDCl}_{3}\right) \delta_{\mathrm{F}}=-$ $117.33\left(\mathrm{dd},{ }^{2} \mathrm{~J}_{\mathrm{F}-\mathrm{H}}=53.3,{ }^{4} \mathrm{~J}_{\mathrm{F}-\mathrm{H}}=1.8 \mathrm{~Hz}, \mathrm{C}_{7} \mathrm{CHF}_{2}\right) \mathrm{ppm} .{ }^{13} \mathrm{C} \mathrm{NMR}$ $\left(101 \mathrm{MHz}, \mathrm{CDCl}_{3}\right) \delta_{\mathrm{c}}=170.31\left(\mathrm{~s}, \mathrm{C}_{9}\right), 151.37\left(\mathrm{t},{ }^{2} \mathrm{~J}_{\mathrm{C}-\mathrm{F}}=23.2 \mathrm{~Hz}\right.$, $\mathrm{C}_{7}$ ), 138.00 (s, $\left.\mathrm{C}_{1}\right), 129.61$ (s, $\left.\mathrm{C}_{3,5}\right), 126.19$ (s, $\left.\mathrm{C}_{4}\right), 124.83$ (s, $\mathrm{C}_{2}$ 6), $109.51\left(\mathrm{t},{ }^{1} \mathrm{~J}_{\mathrm{C}-\mathrm{F}}=241.5 \mathrm{~Hz}, \mathrm{C}_{7} \mathrm{CHF}_{2}\right), 86.73\left(\mathrm{t},{ }^{3} \mathrm{~J}_{\mathrm{C}-\mathrm{F}}=7.0 \mathrm{~Hz}\right.$, $\left.\mathrm{C}_{8}\right), 59.88$ (s, $\left.\mathrm{C}_{9} \mathrm{OCH}_{2} \mathrm{CH}_{3}\right), 14.49$ (s, $\mathrm{C}_{9} \mathrm{OCH}_{2} \mathrm{CH}_{3}$ ). HRMS (ESI positive) for $\mathrm{C}_{12} \mathrm{H}_{14} \mathrm{~F}_{2} \mathrm{NO}_{2}\left[\mathrm{M}^{+}\right]$: calcd 242.0987, found 242.0972 .

\section{Ethyl 3-(phenylamino)but-2-enoate 3b}

a) Ethyl 3-oxobutanoate (1 equiv., $8.14 \mathrm{~mL}, 64.4 \mathrm{mmol}$ ); b) Aniline (1 equiv., $5.87 \mathrm{~mL}, 64.4 \mathrm{mmol}$ ); c) $4 \AA \mathrm{MS}$, for $24 \mathrm{~h}$; d) Ethyl 3(phenylamino)but-2-enoate $3 \mathrm{~b}$ was obtained in $95 \%$ yield $(12.6 \mathrm{~g})$ as a brown oil. ${ }^{1} \mathrm{H}$ NMR $\left(400 \mathrm{MHz}, \mathrm{CDCl}_{3}\right) \delta_{\mathrm{H}}=10.38(\mathrm{~s}, 1 \mathrm{H}, \mathrm{NH})$, $7.36-7.28\left(\mathrm{~m}, 2 \mathrm{H}, \mathrm{C}_{3,5} \mathrm{H}\right), 7.19-7.12\left(\mathrm{~m}, 1 \mathrm{H}, \mathrm{C}_{4} \mathrm{H}\right), 7.11-7.06$ $\left(\mathrm{m}, 2 \mathrm{H}, \mathrm{C}_{2,6} \mathrm{H}\right), 4.69\left(\mathrm{~m}, 1 \mathrm{H}, \mathrm{C}_{8} \mathrm{H}\right), 4.15\left(\mathrm{q},{ }^{3} \mathrm{~J}_{\mathrm{H}-\mathrm{H}}=7.1 \mathrm{~Hz}, 2 \mathrm{H}\right.$, $\left.\mathrm{C}_{9} \mathrm{OCH}_{2} \mathrm{CH}_{3}\right), 2.00\left(\mathrm{~m}, 3 \mathrm{H}, \mathrm{C}_{7} \mathrm{CH}_{3}\right), 1.29\left(\mathrm{t},{ }^{3} \mathrm{~J}_{\mathrm{H}-\mathrm{H}}=7.1 \mathrm{~Hz}, 3 \mathrm{H}\right.$, $\left.\mathrm{C}_{9} \mathrm{OCH}_{2} \mathrm{CH}_{3}\right)$ ppm. ${ }^{13} \mathrm{C}$ NMR $\left(101 \mathrm{MHz}, \mathrm{CDCl}_{3}\right) \delta_{\mathrm{c}}=170.35$ (s, $\left.\mathrm{C}_{9}\right), 158.85$ and 158.83 (d, iso ${ }^{1}-$ iso $\left.^{2}, \mathrm{C}_{7}\right), 139.34$ (s, $\left.\mathrm{C}_{1}\right), 129.03$ $\left(\mathrm{s}, \mathrm{C}_{3}, 5\right), 124.86\left(\mathrm{~s}, \mathrm{C}_{4}\right), 124.34$ and 124.32 (d, iso ${ }^{1}-$ iso $^{2}, \mathrm{C}_{2,6}$ ), $86.10\left(\mathrm{~s}, \mathrm{C}_{8}\right), 58.69$ (s, $\left.\mathrm{C}_{9} \mathrm{OCH}_{2} \mathrm{CH}_{3}\right), 20.25$ and 20.24 (d, iso ${ }^{1}$. iso $\left.{ }^{2}, \mathrm{C}_{7} \mathrm{CH}_{3}\right), 14.57\left(\mathrm{~s}, \mathrm{C}_{9} \mathrm{OCH}_{2} \mathrm{CH}_{3}\right) \mathrm{ppm}$.

Ethyl 3-[(4-chlorophenyl)amino]-4,4-difluorobut-2-enoate 3c a) Ethyl 4,4-difluoroacetoacetate (2 equiv., $10.4 \mathrm{~g}, 8.16 \mathrm{~mL}, 62.3$ mmol); b) p-Chloroaniline (1 equiv., $3.98 \mathrm{~g}, 31.2 \mathrm{mmol}$ ); c) $4 \AA \mathrm{MS}$, for $21 \mathrm{~h}$; d) Ethyl 3-[(4-chlorophenyl)amino]-4,4-difluorobut-2enoate $3 \mathrm{c}$ was obtained in $90 \%$ yield $(7.76 \mathrm{~g})$ as a brown oil with an estimated purity around $75 \%$. ${ }^{1} \mathrm{H} \mathrm{NMR}\left(400 \mathrm{MHz}, \mathrm{CDCl}_{3}\right) \delta_{\mathrm{H}}=$ $9.85(\mathrm{~s}, 1 \mathrm{H}, \mathrm{NH}), 7.31\left(\mathrm{~d},{ }^{3} \mathrm{~J}_{\mathrm{H}-\mathrm{H}}=8.7 \mathrm{~Hz}, 2 \mathrm{H}, \mathrm{C}_{3}, 5 \mathrm{H}\right), 7.09\left(\mathrm{~d},{ }^{3} \mathrm{~J}_{\mathrm{H}}\right.$ $\left.H=8.6 \mathrm{~Hz}, 2 \mathrm{H}, \mathrm{C}_{2,6} \mathrm{H}\right), 6.15\left(\mathrm{t},{ }^{2} \mathrm{~J}_{\mathrm{H}-\mathrm{F}}=53.3 \mathrm{~Hz}, 1 \mathrm{H}, \mathrm{C}_{7} \mathrm{CHF}_{2}\right), 5.25$ $\left(\mathrm{s}, 1 \mathrm{H}, \mathrm{C}_{8} \mathrm{H}\right), 4.20\left(\mathrm{q},{ }^{3} \mathrm{~J}_{\mathrm{H}-\mathrm{H}}=7.1 \mathrm{~Hz}, 2 \mathrm{H}, \mathrm{C}_{9} \mathrm{OCH}_{2} \mathrm{CH}_{3}\right), 1.31\left(\mathrm{t},{ }^{3} \mathrm{~J}_{\mathrm{H}-}\right.$ $\left.\mathrm{H}=7.1 \mathrm{~Hz}, 3 \mathrm{H}, \mathrm{C}_{9} \mathrm{OCH}_{2} \mathrm{CH}_{3}\right) \mathrm{ppm} .{ }^{19} \mathrm{~F} \mathrm{NMR}\left(376 \mathrm{MHz}, \mathrm{CDCl}_{3}\right) \delta_{\mathrm{F}}$ $=-116.93\left(\mathrm{dd},{ }^{2} J_{F-H}=53.3,{ }^{4} J_{F-H}=1.2 \mathrm{~Hz}, \mathrm{C}_{7} \mathrm{CHF}_{2}\right) \mathrm{ppm} .{ }^{13} \mathrm{C} \mathrm{NMR}$ $\left(101 \mathrm{MHz}, \mathrm{CDCl}_{3}\right) \delta_{\mathrm{c}}=170.22\left(\mathrm{~s}, \mathrm{C}_{9}\right), 150.88\left(\mathrm{t},{ }^{2} \mathrm{~J}_{\mathrm{C}-\mathrm{F}}=22.9 \mathrm{~Hz}\right.$, $\left.\mathrm{C}_{7}\right), 136.71$ (s, $\left.\mathrm{C}_{4}\right), 131.80$ (s, $\left.\mathrm{C}_{1}\right), 129.70\left(\mathrm{~s}, \mathrm{C}_{3}, 5\right), 126.15$ (s, $\mathrm{C}_{2}$ 6), $109.81\left(\mathrm{t},{ }^{1} J_{C-F}=242.0 \mathrm{~Hz}, \mathrm{C}_{7} \mathrm{CHF}_{2}\right), 87.87\left(\mathrm{t},{ }^{3} \mathrm{~J}_{\mathrm{C}-\mathrm{F}}=7.2 \mathrm{~Hz}\right.$, $\left.\mathrm{C}_{8}\right), 60.06$ (s, $\left.\mathrm{C}_{9} \mathrm{OCH}_{2} \mathrm{CH}_{3}\right), 14.47$ (s, $\left.\mathrm{C}_{9} \mathrm{OCH}_{2} \mathrm{CH}_{3}\right)$ ppm. HRMS (ESI positive) for $\mathrm{C}_{12} \mathrm{H}_{13} \mathrm{ClF}_{2} \mathrm{NO}_{2}\left[\mathrm{M}^{+}\right]$: calcd 276.0597, found 276.0605 .

\section{Ethyl 4,4,4-trifluoro-3-(phenylamino)but-2-enoate $3 \mathrm{~d}^{[13]}$}

A mixture of ethyl 4,4,4-trifluoroacetoacetate (1 equiv., $1.58 \mathrm{~mL}$, $10.7 \mathrm{mmol}$ ) and aniline (1 equiv., $0.98 \mathrm{~mL}, 10.7 \mathrm{mmol}$ ) was heated at reflux in acetic acid (1 equiv., $0.615 \mathrm{~mL}, 10.7 \mathrm{mmol}$ ) for $3 \mathrm{~h}$. The reaction mixture was quenched by addition of a saturated solution of sodium bicarbonate $\left(\mathrm{NaHCO}_{3}\right)$, extracted with ether, dried over $\mathrm{Na}_{2} \mathrm{SO}_{4}$, and concentrated under vacuum. The crude mixture was purified by column chromatography, using a gradient of AcOEt in pentane (2-98\%) to provide ethyl 4,4,4-trifluoro-3(phenylamino)but-2-enoate $\mathbf{3 d}$ as a colourless oil in $19 \%$ yield $(530 \mathrm{mg}$ ). Both imine and enamine forms of the product were observed by NMR analysis, however the imine form rapidly evolved into the enamine form. Enamine form ${ }^{1} \mathrm{H}$ NMR $(400 \mathrm{MHz}$, $\left.\mathrm{CDCl}_{3}\right) \delta_{\mathrm{H}}=9.84(\mathrm{~s}, 1 \mathrm{H}, \mathrm{NH}), 7.37-7.30\left(\mathrm{~m}, 2 \mathrm{H}, \mathrm{C}_{3,5} \mathrm{H}\right), 7.28-$ $7.22\left(\mathrm{~m}, 1 \mathrm{H}, \mathrm{C}_{4} \mathrm{H}\right), 7.21-7.17\left(\mathrm{~m}, 2 \mathrm{H}, \mathrm{C}_{2,6} \mathrm{H}\right), 5.35\left(\mathrm{~s}, 1 \mathrm{H}, \mathrm{C}_{8} \mathrm{H}\right)$, $4.22\left(\mathrm{q},{ }^{3} \mathrm{~J}_{\mathrm{H}-\mathrm{H}}=7.1 \mathrm{~Hz}, 2 \mathrm{H}, \mathrm{C}_{9} \mathrm{OCH}_{2} \mathrm{CH}_{3}\right), 1.32\left(\mathrm{t},{ }^{3} \mathrm{~J}_{\mathrm{H}-\mathrm{H}}=7.1 \mathrm{~Hz}\right.$, $\left.3 \mathrm{H}, \mathrm{C}_{9} \mathrm{OCH}_{2} \mathrm{CH}_{3}\right) \mathrm{ppm} .{ }^{19} \mathrm{~F} \mathrm{NMR}\left(376 \mathrm{MHz}, \mathrm{CDCl}_{3}\right) \delta_{\mathrm{F}}=-63.33(\mathrm{~s}$, $\left.\mathrm{C}_{7} \mathrm{CF}_{3}\right)$ ppm. ${ }^{13} \mathrm{C}$ NMR $\left(101 \mathrm{MHz}, \mathrm{CDCl}_{3}\right) \delta_{\mathrm{c}}=169.81$ (s, $\left.\mathrm{C}_{9}\right)$, 147.29 (q, $\left.{ }^{2} J_{C-F}=31.4 \mathrm{~Hz}, \mathrm{C}_{7}\right), 138.50\left(\mathrm{~s}, \mathrm{C}_{1}\right), 129.08\left(\mathrm{~s}, \mathrm{C}_{3,5}\right)$, $126.76\left(\mathrm{~s}, \mathrm{C}_{4}\right), 126.13\left(\mathrm{q},{ }^{5} \mathrm{~J}_{\mathrm{C}-\mathrm{F}}=1.5 \mathrm{~Hz}, \mathrm{C}_{2,6}\right), 120.37$ (q, ${ }^{1} \mathrm{~J}_{\mathrm{C}-\mathrm{F}}=$ $277.4 \mathrm{~Hz}, \mathrm{C}_{7} \mathrm{CF}_{3}$ ), 88.67 (q, $\left.{ }^{3} \mathrm{~J}_{\mathrm{C}-\mathrm{F}}=5.4 \mathrm{~Hz}, \mathrm{C}_{8}\right), 60.26(\mathrm{~s}$, $\mathrm{C}_{9} \mathrm{OCH}_{2} \mathrm{CH}_{3}$ ), 14.44 (s, $\mathrm{C}_{9} \mathrm{OCH}_{2} \mathrm{CH}_{3}$ ) ppm. Imine form ${ }^{1} \mathrm{H}$ NMR $\left(400 \mathrm{MHz}, \mathrm{CDCl}_{3}\right) \delta_{\mathrm{H}}=7.40-7.32\left(\mathrm{~m}, 2 \mathrm{H}, \mathrm{C}_{3},{ }_{5} \mathrm{H}\right), 7.20-7.14$ $\left(\mathrm{m}, 1 \mathrm{H}, \mathrm{C}_{4} \mathrm{H}\right), 6.88-6.82\left(\mathrm{~m}, 2 \mathrm{H}, \mathrm{C}_{2,6} \mathrm{H}\right), 4.15\left(\mathrm{q},{ }^{3} \mathrm{~J}_{\mathrm{H}-\mathrm{H}}=7.0 \mathrm{~Hz}\right.$, $\left.2 \mathrm{H}, \mathrm{C}_{9} \mathrm{OCH}_{2} \mathrm{CH}_{3}\right), 3.41\left(\mathrm{~s}, 2 \mathrm{H}, \mathrm{C}_{8} \mathrm{H}\right), 1.24\left(\mathrm{t},{ }^{3} \mathrm{~J}_{\mathrm{H}-\mathrm{H}}=7.1 \mathrm{~Hz}, 3 \mathrm{H}\right.$, $\left.\mathrm{C}_{9} \mathrm{OCH}_{2} \mathrm{CH}_{3}\right) \mathrm{ppm} .{ }^{19} \mathrm{~F} \mathrm{NMR}\left(376 \mathrm{MHz}, \mathrm{CDCl}_{3}\right) \delta_{\mathrm{F}}=-72.64(\mathrm{~s}$, $\left.\mathrm{C}_{7} \mathrm{CF}_{3}\right) \mathrm{ppm}$.

\section{QUINOLINE SYNTHESIS}

\section{Typical procedure $B$ for the synthesis of quinoline derivatives (5)}

Under an argon atmosphere, a solution of the desired FAR (1,1,2,2-tetrafluoro- $N, N$-dimethylethan-1-amine (1A; TFEDMA), 2-chloro- $N, N$-diethyl-1,1,2-trifluoroethan-1-amine (1B; Yarovenko reagent), and $N, N$-diethyl-1,1,2,3,3,3-hexafluoropropan-1-amine (1C; Ishikawa reagent) (1.2 equiv.) was activated by adding boron trifluoride diethyl etherate $\left(\mathrm{BF}_{3} \bullet \mathrm{Et}_{2} \mathrm{O}\right)(1.2$ equiv.) in dry $\mathrm{MeCN}$ (3.6 mmol/5 mL) and stirred for $15 \mathrm{~min}$. Then a solution of the desired enamine derivative (3) (1 equiv.) in dry MeCN (3 mmol/5 $\mathrm{mL}$ ) was slowly syringed into this mixture. After $15 \mathrm{~min}$ at room temperature, the mixture was heated at $50^{\circ} \mathrm{C}$ for $19 \mathrm{~h}$. MeCN was removed under reduced pressure and the crude material was purified by flash chromatography using a gradient of AcOEt in cyclohexane to provide the final compound (5).

The following experiments were carried out according to Typical Procedure $B$, and specific details are reported as: a) FAR (1) and $\mathrm{BF}_{3} \bullet \mathrm{Et}_{2} \mathrm{O}$, or activated FAR (2); b) Enamine (3); and c) yield and aspect. Individual analysis for each compound (5) is given below. Atoms are numbered in the description of NMR spectra according to the Supporting Information.

\section{Ethyl 2,4-bis(difluoromethyl)quinoline-3-carboxylate 5aA}

a) TFEDMA $1 \mathrm{~A}$ (1.2 equiv., $3.26 \mathrm{~mL}, 27.9 \mathrm{mmol}), \mathrm{BF}_{3} \bullet \mathrm{Et}_{2} \mathrm{O}(1.2$ equiv., $3.53 \mathrm{~mL}, 27.9 \mathrm{mmol})$; b) Ethyl 4,4-difluoro-3(phenylamino)but-2-enoate 3a (1 equiv., $5.6 \mathrm{~g}, 23.2 \mathrm{mmol}$ ); c) After purification by flash chromatography using a gradient of AcOEt in cyclohexane (0-5\%), ethyl 2,4bis(difluoromethyl)quinoline-3-carboxylate 5 aA was obtained as a colourless solid in $89 \%$ yield $(6.54 \mathrm{~g}) .{ }^{1} \mathrm{H} \mathrm{NMR}\left(400 \mathrm{MHz}, \mathrm{CDCl}_{3}\right)$ $\delta_{\mathrm{H}}=8.40\left(\mathrm{dd},{ }^{3} J_{\mathrm{H}-\mathrm{H}}=8.6,{ }^{5} \mathrm{~J}_{\mathrm{H}-\mathrm{F}}=1.4 \mathrm{~Hz}, 1 \mathrm{H}, \mathrm{C}_{5} \mathrm{H}\right), 8.23\left(\mathrm{~d},{ }^{3} \mathrm{~J}_{\mathrm{H}-\mathrm{H}}=\right.$ $8.4 \mathrm{~Hz}, 1 \mathrm{H}, \mathrm{C}_{8} \mathrm{H}$ ), 7.88 (ddd, ${ }^{3} \mathrm{~J}_{\mathrm{H}-\mathrm{H} 1}=8.4,{ }^{3} \mathrm{~J}_{\mathrm{H}-\mathrm{H} 4}=7.0,{ }^{4} \mathrm{~J}_{\mathrm{H}-\mathrm{H} 2}=1.2$ 
$\mathrm{Hz}, 1 \mathrm{H}, \mathrm{C}_{6} \mathrm{H}$ ), $7.76\left(\mathrm{ddd},{ }^{3} \mathrm{~J}_{\mathrm{H}-\mathrm{H} 2}=8.3,{ }^{3} \mathrm{~J}_{\mathrm{H}-\mathrm{H} 3}=7.0,{ }^{4} \mathrm{~J}_{\mathrm{H}-\mathrm{H} 1}=1.0 \mathrm{~Hz}\right.$, $\left.1 \mathrm{H}, \mathrm{C}_{7} \mathrm{H}\right), 7.19\left(\mathrm{t},{ }^{2} \mathrm{~J}_{\mathrm{H}-\mathrm{F}}=52.9 \mathrm{~Hz}, 1 \mathrm{H}, \mathrm{C}_{4} \mathrm{CHF}_{2}\right), 6.93\left(\mathrm{t},{ }^{2} \mathrm{~J}_{\mathrm{H}-\mathrm{F}}=\right.$ $54.6 \mathrm{~Hz}, 1 \mathrm{H}, \mathrm{C}_{2} \mathrm{CHF}_{2}$ ), 4.53 (q, $\left.{ }^{3} \mathrm{JH}_{\mathrm{H}-\mathrm{H}}=7.2 \mathrm{~Hz}, 2 \mathrm{H}, \mathrm{C}_{3} \mathrm{OCH}_{2} \mathrm{CH}_{3}\right)$, $1.44\left(\mathrm{t},{ }^{3} \mathrm{JH}_{\mathrm{H}}=7.2 \mathrm{~Hz}, 3 \mathrm{H}, \mathrm{C}_{3} \mathrm{OCH}_{2} \mathrm{CH}_{3}\right) \mathrm{ppm} .{ }^{19} \mathrm{~F} \mathrm{NMR}(376 \mathrm{MHz}$, $\left.\mathrm{CDCl}_{3}\right) \delta_{\mathrm{F}}=-109.64\left(\mathrm{dd},{ }^{2} \mathrm{~J}_{F-H}=53.0,{ }^{5} \mathrm{~J}_{F-H}=2.0 \mathrm{~Hz}, \mathrm{C}_{4} \mathrm{CHF}_{2}\right)$, $115.25\left(\mathrm{~d},{ }^{2} \mathrm{~J}_{F-H}=54.6 \mathrm{~Hz}, \mathrm{C}_{2} \mathrm{CHF}_{2}\right) \mathrm{ppm} .{ }^{13} \mathrm{C} \mathrm{NMR}(101 \mathrm{MHz}$, $\left.\mathrm{CDCl}_{3}\right) \delta_{\mathrm{C}}=165.32\left(\mathrm{~s}, \mathrm{C}_{3} \mathrm{CO}\right), 148.26\left(\mathrm{t},{ }^{2} \mathrm{~J}_{\mathrm{C}-\mathrm{F}}=25.3 \mathrm{~Hz}, \mathrm{C}_{2}\right)$, $147.56\left(\mathrm{~s}, \mathrm{C}_{10}\right), 137.17\left(\mathrm{t},{ }^{2} \mathrm{~J}_{\mathrm{C}-\mathrm{F}}=23.3 \mathrm{~Hz}, \mathrm{C}_{4}\right), 131.84\left(\mathrm{~s}, \mathrm{C}_{6}\right)$, 130.80 (s, C 8 ), 129.99 (s, $\left.\mathrm{C}_{7}\right), 125.43\left(\mathrm{t},{ }^{4} \mathrm{~J}_{\mathrm{C}-F}=3.7 \mathrm{~Hz}, \mathrm{C}_{5}\right), 124.21$ $\left(\mathrm{t},{ }^{3} J_{C-F}=6.6 \mathrm{~Hz}, \mathrm{C}_{3}\right), 123.57\left(\mathrm{~s}, \mathrm{C}_{9}\right), 114.08\left(\mathrm{t},{ }^{1} \mathrm{~J}_{\mathrm{C}-\mathrm{F}}=242.4 \mathrm{~Hz}\right.$, $\mathrm{C}_{2} \mathrm{CHF}_{2}$ ), 112.93 (t, $\left.{ }^{1} \mathrm{~J}_{\mathrm{C}-\mathrm{F}}=241.4 \mathrm{~Hz}, \mathrm{C}_{4} \mathrm{CHF}_{2}\right), 63.30(\mathrm{~s}$, $\left.\mathrm{C}_{3} \mathrm{OCH}_{2} \mathrm{CH}_{3}\right), 14.03$ (s, $\left.\mathrm{C}_{3} \mathrm{OCH}_{2} \mathrm{CH}_{3}\right)$ ppm. IR v $\left(\mathrm{cm}^{-1}\right): 2988-2901$ $\left(\mathrm{C}_{\mathrm{sp} 3} \mathrm{H}\right), 1721$ ( $\left.\mathrm{C}=\mathrm{O}_{\text {ester }}\right) . \mathrm{C}_{14} \mathrm{H}_{11} \mathrm{~F}_{4} \mathrm{NO}_{2}$ (301): calcd (\%) N 4.65, C 55.82, H 3.68, found N 4.63, C 55.86, H 3.78. MP: $65.5-66.7^{\circ} \mathrm{C}$.

\section{Ethyl 4-(chlorofluoromethyl)-2-(difluoromethyl)quinoline-3- carboxylate 5aB'}

The required FAR 1B' was freshly prepared and activated by $\mathrm{BF}_{3} \bullet \mathrm{Et}_{2} \mathrm{O}$, in order to be used as the corresponding fluoroiminium salt 2B'.

a) $\mathrm{N}$-(2-Chloro-1,2-difluoroethylidene)- $\mathrm{N}$-methylmethanaminium tetrafluoroborate 2B' (1.30 equiv., $1.19 \mathrm{~g}, 5.20 \mathrm{mmol})$; b) Ethyl 4,4-difluoro-3-(phenylamino)but-2-enoate 3a (1 equiv., $1.56 \mathrm{~g}$, $3.87 \mathrm{mmol}$ ); c) After purification by flash chromatography using a gradient of AcOEt in cyclohexane (0-5\%), ethyl 4(chlorofluoromethyl)-2-(difluoromethyl)quinoline-3-carboxylate 5aB' was obtained as a yellow oil in $85 \%$ yield $(1.04 \mathrm{~g}) .{ }^{1} \mathrm{H}$ NMR $\left(400 \mathrm{MHz}, \mathrm{CDCl}_{3}\right) \delta_{\mathrm{H}}=8.48\left(\mathrm{~d},{ }^{3} \mathrm{~J}_{\mathrm{H}-\mathrm{H}}=8.6 \mathrm{~Hz}, 1 \mathrm{H}, \mathrm{C}_{5} \mathrm{H}\right), 8.23(\mathrm{~d}$, $\left.{ }^{3} J_{H-H}=7.9 \mathrm{~Hz}, 1 \mathrm{H}, \mathrm{C} 8 \mathrm{H}\right), 7.88\left(\mathrm{ddd},{ }^{3} \mathrm{~J}_{\mathrm{H}-\mathrm{H} 1}=8.4,{ }^{3} \mathrm{~J}_{\mathrm{H}-\mathrm{H} 4}=6.9,{ }^{4} \mathrm{~J}_{\mathrm{H}-\mathrm{H} 2}\right.$ $=1.3 \mathrm{~Hz}, 1 \mathrm{H}, \mathrm{C}_{6} \mathrm{H}$ ), 7.77 (ddd, ${ }^{3} \mathrm{~J}_{\mathrm{H}-\mathrm{H} 2}=8.4,{ }^{3} \mathrm{~J}_{\mathrm{H}-\mathrm{H} 3}=6.9,{ }^{4} \mathrm{~J}_{\mathrm{H}-\mathrm{H} 1}=1.3$ $\left.\mathrm{Hz}, 1 \mathrm{H}, \mathrm{C}_{7} \mathrm{H}\right), 7.62\left(\mathrm{~d},{ }^{2} \mathrm{~J}_{\mathrm{H}-\mathrm{F}}=48.6 \mathrm{~Hz}, 1 \mathrm{H}, \mathrm{C}_{4} \mathrm{CHFCl}\right), 6.93\left(\mathrm{t},{ }^{2} \mathrm{~J}_{\mathrm{H}-}\right.$ $\left.F=54.6 \mathrm{~Hz}, 1 \mathrm{H}, \mathrm{C}_{2} \mathrm{CHF}_{2}\right), 4.61-4.44\left(\mathrm{~m}, 2 \mathrm{H}, \mathrm{C}_{3} \mathrm{OCH}_{2} \mathrm{CH}_{3}\right), 1.45$ $\left(\mathrm{t},{ }^{3} \mathrm{JH}_{\mathrm{H}-\mathrm{H}}=7.2 \mathrm{~Hz}, 3 \mathrm{H}, \mathrm{C}_{3} \mathrm{OCH}_{2} \mathrm{CH}_{3}\right)$ ppm. ${ }^{19} \mathrm{~F} \mathrm{NMR}(376 \mathrm{MHz}$, $\left.\mathrm{CDCl}_{3}\right) \delta_{F}=-115.30\left(\mathrm{~d},{ }^{2} \mathrm{JF}_{F-H}=54.6 \mathrm{~Hz}, \mathrm{C}_{2} \mathrm{CHF}_{2}\right),-133.91\left(\mathrm{~d},{ }^{2} \mathrm{~J}_{F-}\right.$ $\left.H=48.6 \mathrm{~Hz}, \mathrm{C}_{4} \mathrm{CHFCl}\right) \mathrm{ppm} .{ }^{13} \mathrm{C} \mathrm{NMR}\left(101 \mathrm{MHz}, \mathrm{CDCl}_{3}\right) \delta_{\mathrm{c}}=$ 165.40 (s, $\mathrm{C}_{3} \mathrm{CO}$ ), $148.30\left(\mathrm{t},{ }^{2} J_{\mathrm{C}-F}=25.2 \mathrm{~Hz}, \mathrm{C}_{2}\right), 147.71\left(\mathrm{~s}, \mathrm{C}_{10}\right)$, $140.20\left(\mathrm{~d},{ }^{2} \mathrm{~J}_{\mathrm{C}-\mathrm{F}}=21.2 \mathrm{~Hz}, \mathrm{C}_{4}\right), 131.77\left(\mathrm{~s}, \mathrm{C}_{6}\right), 131.02\left(\mathrm{~s}, \mathrm{C}_{8}\right)$, $129.71\left(\mathrm{~s}, \mathrm{C}_{7}\right), 125.43\left(\mathrm{~d},{ }^{4} \mathrm{~J}_{\mathrm{C}-\mathrm{F}}=5.7 \mathrm{~Hz}, \mathrm{C}_{5}\right), 123.07\left(\mathrm{~s}, \mathrm{C}_{9}\right)$, $122.10\left(\mathrm{~d},{ }^{3} \mathrm{~J}_{\mathrm{C}-\mathrm{F}}=5.3 \mathrm{~Hz}, \mathrm{C}_{3}\right), 114.03\left(\mathrm{t},{ }^{1} \mathrm{~J}_{\mathrm{C}-\mathrm{F}}=244.1 \mathrm{~Hz}, \mathrm{C}_{2} \mathrm{CHF}_{2}\right)$, $96.76\left(\mathrm{~d},{ }^{1} \mathrm{~J}_{\mathrm{C}-\mathrm{F}}=244.6 \mathrm{~Hz}, \mathrm{C}_{4} \mathrm{CHFCl}\right), 63.26\left(\mathrm{~s}, \mathrm{C}_{3} \mathrm{OCH}_{2} \mathrm{CH}_{3}\right)$, 14.04 (s, $\left.\mathrm{C}_{3} \mathrm{OCH}_{2} \mathrm{CH}_{3}\right)$ ppm. IR v $\left(\mathrm{cm}^{-1}\right):$ 2987-2907 $\left(\mathrm{C}_{\mathrm{sp} 3} \mathrm{H}\right), 1727$ (C=Oester). $\mathrm{C}_{14} \mathrm{H}_{11} \mathrm{~F}_{3} \mathrm{CINO}_{2}$ (317): calcd (\%) N 4.41, C 52.93, H 3.49, found N 4.32, C 52.63, H 3.59.

\section{Ethyl}

2-(difluoromethyl)-4(fluoro(trifluoromethoxy)methyl)quinoline-3-carboxylate 5aD a) $\quad \mathrm{N}$-(1,2-Difluoro-2-(trifluoromethoxy)ethylidene)- $\mathrm{N}$ methylmethanaminium tetrafluoroborate 2D (1.57 equiv., $1.7 \mathrm{~g}$, $6.1 \mathrm{mmol}$ ); b) Ethyl 4,4-difluoro-3-(phenylamino)but-2-enoate 3a (1 equiv., $1.56 \mathrm{~g}, 3.87 \mathrm{mmol}$ ); c) After purification by flash chromatography using a gradient of AcOEt in cyclohexane (0$20 \%$ ), ethyl 2-(difluoromethyl)-4(fluoro(trifluoromethoxy)methyl)quinoline-3-carboxylate 5aD was obtained as a colourless solid in $97 \%$ yield $(1.38 \mathrm{~g}) .{ }^{1} \mathrm{H}$ NMR (400 $\left.\mathrm{MHz}, \mathrm{CDCl}_{3}\right) \delta_{\mathrm{H}}=8.41\left(\mathrm{~d},{ }^{3} \mathrm{H}_{\mathrm{H}-\mathrm{H}}=8.6 \mathrm{~Hz}, 1 \mathrm{H}, \mathrm{C}_{5} \mathrm{H}\right), 8.23\left(\mathrm{~d},{ }^{3} \mathrm{~J}_{\mathrm{H}-\mathrm{H}}\right.$ $\left.=8.4 \mathrm{~Hz}, 1 \mathrm{H}, \mathrm{C}_{8} \mathrm{H}\right), 7.93-7.82\left(\mathrm{~m}, 1 \mathrm{H}, \mathrm{C}_{7} \mathrm{H}\right), 7.82-7.67(\mathrm{~m}, 1 \mathrm{H}$,
$\left.\mathrm{C}_{6} \mathrm{H}\right), 7.28\left(\mathrm{~d},{ }^{2} \mathrm{~J}_{\mathrm{H}-\mathrm{F}}=54.8 \mathrm{~Hz}, 1 \mathrm{H}, \mathrm{C}_{4} \mathrm{CHFOCF}_{3}\right), 6.95\left(\mathrm{t},{ }^{2} \mathrm{~J}_{\mathrm{H}-\mathrm{F}}=\right.$ $\left.54.6 \mathrm{~Hz}, 1 \mathrm{H}, \mathrm{C}_{2} \mathrm{CHF}_{2}\right), 4.61-4.45\left(\mathrm{~m}, 2 \mathrm{H}, \mathrm{C}_{3} \mathrm{OCH}_{2} \mathrm{CH}_{3}\right), 1.44(\mathrm{t}$, $\left.{ }^{3} \mathrm{JH}_{\mathrm{H}}=7.2 \mathrm{~Hz}, 3 \mathrm{H}, \mathrm{C}_{3} \mathrm{OCH}_{2} \mathrm{CH}_{3}\right) \mathrm{ppm} .{ }^{19} \mathrm{~F} \mathrm{NMR}\left(376 \mathrm{MHz}, \mathrm{CDCl}_{3}\right)$ $\delta_{\mathrm{F}}=-59.39\left(\mathrm{~d},{ }^{4} \mathrm{JF}_{\mathrm{F}-\mathrm{F}}=4.9 \mathrm{~Hz}, \mathrm{C}_{4} \mathrm{CHFOCF}_{3}\right),-114.33--116.46(\mathrm{~m}$, $\left.\mathrm{A}_{2} \mathrm{~B}_{2}, \Delta \mathrm{v}=485.13 \mathrm{~Hz}, \mathrm{C}_{2} \mathrm{CHF}_{2}\right),-120.55\left(\mathrm{dqd},{ }^{2} \mathrm{~J}_{F-H}=54.9,{ }^{4} \mathrm{~J}_{F-F}=\right.$ $\left.4.8,{ }^{5} J_{F-H}=2.1 \mathrm{~Hz}, \mathrm{C}_{4} \mathrm{CHFOCF}_{3}\right) \mathrm{ppm} .{ }^{13} \mathrm{C} \mathrm{NMR}\left(101 \mathrm{MHz}, \mathrm{CDCl}_{3}\right)$ $\delta_{\mathrm{C}}=165.28\left(\mathrm{~s}, \mathrm{C}_{3} \mathrm{CO}\right), 148.35\left(\mathrm{t},{ }^{2} \mathrm{~J}_{\mathrm{C}-\mathrm{F}}=25.3 \mathrm{~Hz}, \mathrm{C}_{2}\right), 147.68(\mathrm{~s}$, $\left.\mathrm{C}_{9}\right), 136.42\left(\mathrm{~d},{ }^{2} \mathrm{~J}_{\mathrm{C}-\mathrm{F}}=24.1 \mathrm{~Hz}, \mathrm{C}_{4}\right), 131.91\left(\mathrm{~s}, \mathrm{C}_{7}\right), 130.87\left(\mathrm{~s}, \mathrm{C}_{8}\right)$, 130.02 (s, C6), $125.63\left(\mathrm{~d},{ }^{4} \mathrm{~J}_{\mathrm{C}-F}=4.9 \mathrm{~Hz}, \mathrm{C}_{5}\right), 123.97\left(\mathrm{~d},{ }^{3} \mathrm{~J}_{\mathrm{C}-\mathrm{F}}=6.2\right.$ $\left.\mathrm{Hz}, \mathrm{C}_{3}\right), 123.46$ (s, $\left.\mathrm{C}_{10}\right), 121.08$ (qd, ${ }^{1} \mathrm{~J}_{\mathrm{C}-F}=262.6,{ }^{3} \mathrm{~J}_{\mathrm{C}-\mathrm{F}}=1.7 \mathrm{~Hz}$, $\mathrm{C}_{4} \mathrm{CHFOCF}_{3}$ ), $114.05\left(\mathrm{t},{ }^{1} \mathrm{~J}_{\mathrm{C}-\mathrm{F}}=244.1 \mathrm{~Hz}, \mathrm{C}_{2} \mathrm{CHF}_{2}\right.$ ), 103.11 (dq, $\left.{ }^{1} J_{C-F}=232.2,{ }^{3} J_{C-F}=3.9 \mathrm{~Hz}, \mathrm{C}_{4} \mathrm{CHFOCF}_{3}\right), 63.42\left(\mathrm{~s}, \mathrm{C}_{3} \mathrm{OCH}_{2} \mathrm{CH}_{3}\right)$, $13.90\left(\mathrm{~s}, \mathrm{C}_{3} \mathrm{OCH}_{2} \mathrm{CH}_{3}\right)$ ppm. IR v $\left(\mathrm{cm}^{-1}\right): 2991-2943\left(\mathrm{C}_{\mathrm{sp} 3} \mathrm{H}\right), 1727$ (C=Oester). $\mathrm{C}_{15} \mathrm{H}_{11} \mathrm{~F}_{6} \mathrm{NO}_{3}$ (367): calcd (\%) N 3.81, C 49.06, H 3.02, found N 3.74, C 49.23, H 3.18. MP: $43.3-44.8^{\circ} \mathrm{C}$.

Ethyl 4-(difluoromethyl)-2-methylquinoline-3-carboxylate $5 \mathrm{bA}$

Deviation from the general procedure: $\mathrm{H}_{2} \mathrm{SO}_{4}$ was added after formation of the vinamidine.

a) TFEDMA 1A (1.2 equiv., $0.207 \mathrm{~mL}, 1.77 \mathrm{mmol}), \mathrm{BF}_{3} \bullet \mathrm{Et}_{2} \mathrm{O}(1.2$ equiv., $0.224 \mathrm{~mL}, 1.77 \mathrm{mmol}$ ); b) Ethyl 3-(phenylamino)but-2enoate $3 \mathbf{b}$ (1 equiv., $0.466 \mathrm{~g}, 1.47 \mathrm{mmol}$ ), followed by addition of $\mathrm{H}_{2} \mathrm{SO}_{4}$ (20 equiv., $18 \mathrm{M}, 1.64 \mathrm{~mL}, 29.5 \mathrm{mmol}$ ) after $19 \mathrm{~h}$ and stirring for an additional $12 \mathrm{~h}$; c) Traces of ethyl 4-(difluoromethyl)2-methylquinoline-3-carboxylate $\mathbf{5 b A}$ were obtained after purification using a gradient of AcOEt in cyclohexane $(0-80 \%)$ as a clear yellow oil. ${ }^{1} \mathrm{H}$ NMR $\left(500 \mathrm{MHz}, \mathrm{CDCl}_{3}\right) \delta_{\mathrm{H}}=8.25$ (dd, ${ }^{3} \mathrm{~J}_{\mathrm{H}-\mathrm{H}}$ $\left.=8.5,{ }^{5} J_{H-F}=1.6 \mathrm{~Hz}, 1 \mathrm{H}, \mathrm{C}_{5} \mathrm{H}\right), 8.09\left(\mathrm{~d},{ }^{3} \mathrm{~J}_{\mathrm{H}-\mathrm{H}}=8.4 \mathrm{~Hz}, 1 \mathrm{H}, \mathrm{C}_{8} \mathrm{H}\right)$, $7.79\left(\mathrm{ddd},{ }^{3} \mathrm{~J}_{\mathrm{H}-\mathrm{H} 2}=8.4,{ }^{3} \mathrm{~J}_{\mathrm{H}-\mathrm{H} 4}=6.9, \mathrm{~J}_{\mathrm{H}-\mathrm{H} 1}=1.3 \mathrm{~Hz}, 1 \mathrm{H}, \mathrm{C}_{7} \mathrm{H}\right), 7.63$ (ddd, $\left.{ }^{3} \mathrm{~J}_{\mathrm{H}-\mathrm{H} 1}=8.3,{ }^{3} \mathrm{~J}_{\mathrm{H}-\mathrm{H} 3}=6.9,{ }^{4} \mathrm{JH}_{\mathrm{H}-\mathrm{H} 2}=1.2 \mathrm{~Hz}, 1 \mathrm{H}, \mathrm{C}_{6} \mathrm{H}\right), 7.10(\mathrm{t}$, $\left.{ }^{2} J_{H-F}=53.3 \mathrm{~Hz}, 1 \mathrm{H}, \mathrm{C}_{4} \mathrm{CHF}_{2}\right), 4.51\left(\mathrm{q},{ }^{3} \mathrm{~J}_{\mathrm{H}-\mathrm{H}}=7.2 \mathrm{~Hz}, 2 \mathrm{H}\right.$, $\left.\mathrm{C}_{3} \mathrm{OCH}_{2} \mathrm{CH}_{3}\right), 2.77\left(\mathrm{~s}, 3 \mathrm{H}, \mathrm{C}_{2} \mathrm{CH}_{3}\right), 1.45\left(\mathrm{t},{ }^{3} \mathrm{~J}_{\mathrm{H}-\mathrm{H}}=7.2 \mathrm{~Hz}, 3 \mathrm{H}\right.$, $\left.\mathrm{C}_{3} \mathrm{OCH}_{2} \mathrm{CH}_{3}\right)$ ppm. ${ }^{19} \mathrm{~F} \mathrm{NMR}\left(376 \mathrm{MHz}, \mathrm{CDCl}_{3}\right) \delta_{\mathrm{F}}=-110.31(\mathrm{dd}$, $\left.{ }^{2} J_{F-H}=53.2,{ }^{5} J_{F-H}=1.9 \mathrm{~Hz}, \mathrm{C}_{4} \mathrm{CHF}_{2}\right) \mathrm{ppm} .{ }^{13} \mathrm{C} \mathrm{NMR}(126 \mathrm{MHz}$, $\left.\mathrm{CDCl}_{3}\right) \delta_{\mathrm{c}}=167.51\left(\mathrm{~s}, \mathrm{C}_{3} \mathrm{CO}\right), 154.78\left(\mathrm{~s}, \mathrm{C}_{2}\right), 148.29\left(\mathrm{~s}, \mathrm{C}_{10}\right)$, $134.78\left(\mathrm{t},{ }^{2} \mathrm{~J}_{\mathrm{C}-\mathrm{F}}=22.6 \mathrm{~Hz}, \mathrm{C}_{4}\right), 130.97\left(\mathrm{~s}, \mathrm{C}_{7}\right), 129.72\left(\mathrm{~s}, \mathrm{C}_{8}\right)$, $127.73\left(\mathrm{~s}, \mathrm{C}_{6}\right), 126.54\left(\mathrm{t},{ }^{3} \mathrm{~J}_{\mathrm{C}-F}=6.0 \mathrm{~Hz}, \mathrm{C}_{3}\right), 124.79\left(\mathrm{t},{ }^{4} \mathrm{~J}_{\mathrm{C}-F}=2.9\right.$ $\mathrm{Hz}, \mathrm{C}_{5}$ ), 121.72 (s, C9), 113.38 (t, ${ }^{1} \mathrm{~J}_{\mathrm{C}-\mathrm{F}}=241.2 \mathrm{~Hz}, \mathrm{C}_{4} \mathrm{CHF}_{2}$ ), 62.67 (s, $\mathrm{C}_{3} \mathrm{OCH}_{2} \mathrm{CH}_{3}$ ), 23.83 (s, $\left.\mathrm{C}_{2} \mathrm{CH}_{3}\right), 14.26\left(\mathrm{~s}, \mathrm{C}_{3} \mathrm{OCH}_{2} \mathrm{CH}_{3}\right) \mathrm{ppm}$. IR $\vee\left(\mathrm{cm}^{-1}\right): 2986-2929\left(\mathrm{C}_{\mathrm{sp} 3} \mathrm{H}\right), 1726\left(\mathrm{C}=\mathrm{O}_{\text {ester }}\right) . \mathrm{C}_{14} \mathrm{H}_{13} \mathrm{~F}_{2} \mathrm{NO}_{2}$ (265): calcd (\%) N 5.28, C 63.39, H 4.94, found N 4.89, C 63.62, H 5.21 .

Ethyl 4-[fluoro(trifluoromethoxy)methyl]-2-methylquinoline3-carboxylate 5bD

Deviation from the general procedure: $\mathrm{H}_{2} \mathrm{SO}_{4}$ was added after formation of the vinamidine.

The required FAR 1D was freshly prepared and activated by $\mathrm{BF}_{3} \bullet \mathrm{Et}_{2} \mathrm{O}$, in order to be used as the corresponding fluoroiminium salt 2D.

a) $\quad \mathrm{N}$-(1,2-Difluoro-2-(trifluoromethoxy)ethylidene)- $\mathrm{N}$ methylmethanaminium tetrafluoroborate 2D (1.59 equiv., $1.7 \mathrm{~g}$, $6.1 \mathrm{mmol}$ ); b) Ethyl 3-(phenylamino)but-2-enoate 3b (1 equiv., $1.21 \mathrm{~g}, 3.82 \mathrm{mmol}$ ), followed by addition of $\mathrm{H}_{2} \mathrm{SO}_{4}$ (10 equiv., 18 $\mathrm{M}, 2.12 \mathrm{~mL}, 38.2 \mathrm{mmol}$ ) after $19 \mathrm{~h}$ and stirring for an additional 4 h; c) Ethyl 4-[fluoro(trifluoromethoxy)methyl]-2-methylquinoline-3carboxylate 5bD was obtained after purification using a gradient 
of AcOEt in cyclohexane $(0-20 \%)$ in $27 \%$ yield $(346 \mathrm{mg}$ ) as a light yellow oil. ${ }^{1} \mathrm{H}$ NMR $\left(400 \mathrm{MHz}, \mathrm{CDCl}_{3}\right) \delta_{\mathrm{H}}=8.25\left(\mathrm{~d},{ }^{3} \mathrm{~J}_{\mathrm{H}-\mathrm{H}}=8.6 \mathrm{~Hz}\right.$, $\left.{ }^{5} J_{H-F}=1.5,1 \mathrm{H}, \mathrm{C}_{5} \mathrm{H}\right), 8.09\left(\mathrm{dd},{ }^{3} \mathrm{~J}_{\mathrm{H}-\mathrm{H}}=8.5,{ }^{6} \mathrm{~J}_{\mathrm{H}-\mathrm{F}}=0.6 \mathrm{~Hz}, 1 \mathrm{H}, \mathrm{C}_{8} \mathrm{H}\right)$, 7.79 (ddd, $\left.{ }^{3} \mathrm{~J}_{\mathrm{H}-\mathrm{H} 2}=8.4,{ }^{3} \mathrm{~J}_{\mathrm{H}-\mathrm{H} 4}=6.9,{ }^{4} \mathrm{~J}_{\mathrm{H}-\mathrm{H} 1}=1.3 \mathrm{~Hz}, 1 \mathrm{H}, \mathrm{C}_{7} \mathrm{H}\right), 7.63$ (ddd, $\left.{ }^{3} \mathrm{~J}_{\mathrm{H}-\mathrm{H} 1}=8.4,{ }^{3} \mathrm{~J}_{\mathrm{H}-\mathrm{H} 3}=6.9,{ }^{4} \mathrm{~J}_{\mathrm{H}-\mathrm{H} 2}=1.3 \mathrm{~Hz}, 1 \mathrm{H}, \mathrm{C}_{6} \mathrm{H}\right), 7.16(\mathrm{~d}$, $\left.{ }^{2} J_{H-F}=55.1 \mathrm{~Hz}, 1 \mathrm{H}, \mathrm{C}_{4} \mathrm{CHFOCF}_{3}\right), 4.58-4.44(\mathrm{~m}, 2 \mathrm{H}$, $\left.\mathrm{C}_{3} \mathrm{OCH}_{2} \mathrm{CH}_{3}\right), 2.77\left(\mathrm{~s}, 3 \mathrm{H}, \mathrm{C}_{2} \mathrm{CH}_{3}\right), 1.44\left(\mathrm{t},{ }^{3} \mathrm{~J}_{\mathrm{H}-\mathrm{H}}=7.2 \mathrm{~Hz}, 3 \mathrm{H}\right.$, $\left.\mathrm{C}_{3} \mathrm{OCH}_{2} \mathrm{CH}_{3}\right) \mathrm{ppm} .{ }^{19} \mathrm{~F} \mathrm{NMR}\left(376 \mathrm{MHz}, \mathrm{CDCl}_{3}\right) \delta_{\mathrm{F}}=-59.28\left(\mathrm{~d},{ }^{4} J_{\mathrm{F}}\right.$ $\left.F=5.2 \mathrm{~Hz}, \mathrm{C}_{4} \mathrm{CHFOCF}_{3}\right),-121.02\left(\mathrm{dqd},{ }^{2} \mathrm{~J}_{F-H}=55.1,{ }^{4} \mathrm{~J}_{F-F}=5.0\right.$, $\left.{ }^{5} J_{F-H}=1.9 \mathrm{~Hz}, \mathrm{C}_{4} \mathrm{CHFOCF}_{3}\right) \mathrm{ppm} .{ }^{13} \mathrm{C} \mathrm{NMR}\left(101 \mathrm{MHz}, \mathrm{CDCl}_{3}\right) \delta_{\mathrm{C}}$ $=167.32\left(\mathrm{~s}, \mathrm{C}_{3} \mathrm{CO}\right), 154.81\left(\mathrm{~s}, \mathrm{C}_{2}\right), 148.41\left(\mathrm{~s}, \mathrm{C}_{10}\right), 134.01\left(\mathrm{~d},{ }^{2} \mathrm{~J}_{\mathrm{C}}\right.$ $\left.F=23.4 \mathrm{~Hz}, \mathrm{C}_{4}\right), 131.04\left(\mathrm{~s}, \mathrm{C}_{7}\right), 129.79\left(\mathrm{~s}, \mathrm{C}_{8}\right), 127.77\left(\mathrm{~s}, \mathrm{C}_{6}\right)$, $126.45\left(\mathrm{~d},{ }^{3} \mathrm{~J}_{\mathrm{C}-\mathrm{F}}=5.7 \mathrm{~Hz}, \mathrm{C}_{3}\right), 124.90\left(\mathrm{~d},{ }^{4} \mathrm{~J}_{\mathrm{C}-\mathrm{F}}=3.9 \mathrm{~Hz}, \mathrm{C}_{5}\right), 121.52$ (s, C 9 ), 121.11 (qd, ${ }^{1} J_{C-F}=262.2,{ }^{3} J_{C-F}=1.7 \mathrm{~Hz}, \mathrm{C}_{4} \mathrm{CHFOCF}_{3}$ ), $103.55\left(\mathrm{dq},{ }^{1} \mathrm{~J}_{\mathrm{C}-\mathrm{F}}=232.2,{ }^{3} \mathrm{~J}_{\mathrm{C}-\mathrm{F}}=3.9 \mathrm{~Hz}, \mathrm{C}_{4} \mathrm{CHFOCF}_{3}\right), 62.76(\mathrm{~s}$, $\left.\mathrm{C}_{3} \mathrm{OCH}_{2} \mathrm{CH}_{3}\right), 23.96$ (s, $\left.\mathrm{C}_{2} \mathrm{CH}_{3}\right), 14.12\left(\mathrm{~s}, \mathrm{C}_{3} \mathrm{OCH}_{2} \mathrm{CH}_{3}\right) \mathrm{ppm}$. IR v $\left(\mathrm{cm}^{-1}\right): 2987\left(\mathrm{C}_{\mathrm{sp} 3} \mathrm{H}\right), 1727\left(\mathrm{C}=\mathrm{O}_{\text {ester }}\right)$. HRMS (ESI positive) for $\mathrm{C}_{15} \mathrm{H}_{14} \mathrm{~F}_{4} \mathrm{NO}_{3}[\mathrm{M}+]$ : calcd 332.0904 , found 332.0898 .

\section{Ethyl 6-chloro-2,4-bis(difluoromethyl)quinoline-3-}

\section{carboxylate 5cA}

Deviation from the general procedure: $\mathrm{H}_{2} \mathrm{SO}_{4}$ was added after formation of the vinamidine.

a) TFEDMA 1A (1.2 equiv., $0.58 \mathrm{~mL}, 4.96 \mathrm{mmol}), \mathrm{BF}_{3} \bullet \mathrm{Et}_{2} \mathrm{O}(1.2$ equiv., $0.628 \mathrm{~mL}, 4.96 \mathrm{mmol})$; b) Ethyl 3-[(4-chlorophenyl)amino]4,4-difluorobut-2-enoate 3c (1 equiv., $1.52 \mathrm{~g}, 4.13 \mathrm{mmol}$ ), followed by addition of $\mathrm{H}_{2} \mathrm{SO}_{4}$ (10 equiv., $18 \mathrm{M}, 2.29 \mathrm{~mL}, 41.3$ $\mathrm{mmol}$ ) after $19 \mathrm{~h}$ and stirring for an additional $4 \mathrm{~h}$; c) Ethyl 6chloro-2,4-bis(difluoromethyl)quinoline-3-carboxylate 5cA was obtained after purification using a gradient of AcOEt in cyclohexane $(0-5 \%)$ as a beige solid in $26 \%$ yield $(358 \mathrm{mg}) .{ }^{1} \mathrm{H}$ $\operatorname{NMR}\left(400 \mathrm{MHz}, \mathrm{CDCl}_{3}\right) \delta_{\mathrm{H}}=8.39\left(\mathrm{dd},{ }^{4} \mathrm{~J}_{\mathrm{H}-\mathrm{H}}=3.7,{ }^{5} \mathrm{~J}_{\mathrm{H}-\mathrm{F}}=1.8 \mathrm{~Hz}\right.$, $\left.1 \mathrm{H}, \mathrm{C}_{5} \mathrm{H}\right), 8.17\left(\mathrm{~d},{ }^{3} \mathrm{~J}_{\mathrm{H}-\mathrm{H}}=9.0 \mathrm{~Hz}, 1 \mathrm{H}, \mathrm{C}_{8} \mathrm{H}\right), 7.83\left(\mathrm{dd},{ }^{3} \mathrm{~J}_{\mathrm{H}-\mathrm{H}}=9.0\right.$, $\left.{ }^{4} J_{H-H}=2.2 \mathrm{~Hz}, 1 \mathrm{H}, \mathrm{C}_{7} \mathrm{H}\right), 7.12\left(\mathrm{t},{ }^{2} J_{H-F}=52.0 \mathrm{~Hz}, 1 \mathrm{H}, \mathrm{C}_{4} \mathrm{CHF}_{2}\right)$, $6.91\left(\mathrm{t},{ }^{2} \mathrm{~J}_{\mathrm{H}-\mathrm{F}}=54.5 \mathrm{~Hz}, 1 \mathrm{H}, \mathrm{C}_{2} \mathrm{CHF}_{2}\right), 4.53\left(\mathrm{q},{ }^{3} \mathrm{~J}_{\mathrm{H}-\mathrm{H}}=7.2 \mathrm{~Hz}, 1 \mathrm{H}\right.$, $\left.\mathrm{C}_{3} \mathrm{OCH}_{2} \mathrm{CH}_{3}\right), 1.45\left(\mathrm{t},{ }^{3} \mathrm{~J}_{\mathrm{H}-\mathrm{H}}=7.2 \mathrm{~Hz}, 1 \mathrm{H}, \mathrm{C}_{3} \mathrm{OCH}_{2} \mathrm{CH}_{3}\right) \mathrm{ppm} .{ }^{19} \mathrm{~F}$ NMR (376 MHz, CDCl $\left.{ }_{3}\right) \delta_{F}=-109.66\left(\mathrm{dd},{ }^{2} J_{F-H}=52.8,{ }^{5} J_{F-H}=1.7\right.$ $\left.\mathrm{Hz}, \mathrm{C}_{4} \mathrm{CHF}_{2}\right),-115.46\left(\mathrm{~d},{ }^{2} \mathrm{JF}_{\mathrm{F}-\mathrm{H}}=54.5 \mathrm{~Hz}, \mathrm{C}_{2} \mathrm{CHF}_{2}\right) \mathrm{ppm} .{ }^{13} \mathrm{C} \mathrm{NMR}$ $\left(101 \mathrm{MHz}, \mathrm{CDCl}_{3}\right) \delta_{\mathrm{c}}=164.91\left(\mathrm{~s}, \mathrm{C}_{3} \mathrm{CO}\right), 148.53\left(\mathrm{t},{ }^{2} \mathrm{~J}_{\mathrm{C}-\mathrm{F}}=25.5\right.$ $\left.\mathrm{Hz}, \mathrm{C}_{2}\right), 145.99\left(\mathrm{~s}, \mathrm{C}_{6}\right), 137.61-135.84\left(\mathrm{~m}, \mathrm{C}_{4}+\mathrm{C}_{10}\right), 132.99$ (s, $\left.\mathrm{C}_{7}\right), 132.22\left(\mathrm{~s}, \mathrm{C}_{8}\right), 125.09\left(\mathrm{t},{ }^{3} \mathrm{~J}_{\mathrm{C}-F}=6.7 \mathrm{~Hz}, \mathrm{C}_{3}\right), 124.63\left(\mathrm{t},{ }^{4} \mathrm{~J}_{\mathrm{C}-F}=\right.$ $\left.4.2 \mathrm{~Hz}, \mathrm{C}_{5}\right), 124.18\left(\mathrm{~s}, \mathrm{C}_{9}\right), 113.86\left(\mathrm{t},{ }^{1} \mathrm{~J}_{\mathrm{C}-\mathrm{F}}=245.4 \mathrm{~Hz}, \mathrm{C}_{2} \mathrm{CHF}_{2}\right.$ ), $112.69\left(\mathrm{t},{ }^{1} \mathrm{~J}_{\mathrm{C}-\mathrm{F}}=241.5 \mathrm{~Hz}, \mathrm{C}_{4} \mathrm{CHF}_{2}\right), 63.53\left(\mathrm{~s}, \mathrm{C}_{3} \mathrm{OCH}_{2} \mathrm{CH}_{3}\right)$, $14.04\left(\mathrm{~s}, \mathrm{C}_{3} \mathrm{OCH}_{2} \mathrm{CH}_{3}\right)$ ppm. IR v $\left(\mathrm{cm}^{-1}\right): 2990-2941\left(\mathrm{C}_{\mathrm{sp} 3} \mathrm{H}\right), 1721$ (C=Oester). $\mathrm{C}_{14} \mathrm{H}_{10} \mathrm{~F}_{4} \mathrm{NClO}_{2}$ (335): calcd (\%) N 4.17, C 50.09, H 3.00, found N 3.98, C 49.59, H 3.19. MP: $71.9-74.3^{\circ} \mathrm{C}$

\section{Ethyl 4-(difluoromethyl)-2-(trifluoromethyl)quinoline-3- carboxylate $5 \mathrm{dA}$}

a) TFEDMA $1 \mathrm{~A}$ (1.2 equiv., $0.176 \mathrm{~mL}, 1.51 \mathrm{mmol}), \mathrm{BF}_{3} \bullet \mathrm{Et}_{2} \mathrm{O}(1.2$ equiv., $0.191 \mathrm{~mL}, 1.51 \mathrm{mmol}) ;$ b) Ethyl 4,4,4-trifluoro-3(phenylamino)but-2-enoate 3d (1 equiv., $406 \mathrm{mg}, 1.26 \mathrm{mmol}$ ); c) Ethyl 4-(difluoromethyl)-2-(trifluoromethyl)quinoline-3carboxylate 5dA was obtained after purification using a gradient of AcOEt in cyclohexane (0-5\%) as colourless solid in $80 \%$ yield (316 mg). ${ }^{1} \mathrm{H}$ NMR $\left(400 \mathrm{MHz}, \mathrm{CDCl}_{3}\right) \delta_{\mathrm{H}}=8.44$ (dd, ${ }^{3} \mathrm{~J}_{\mathrm{H}-\mathrm{H}}=8.6$, $\left.{ }^{5} J_{H-F}=2.0 \mathrm{~Hz}, 1 \mathrm{H}, \mathrm{C}_{5} \mathrm{H}\right), 8.31\left(\mathrm{dd},{ }^{3} \mathrm{~J}_{\mathrm{H}-\mathrm{H}}=8.5,{ }^{6} \mathrm{~J}_{\mathrm{H}-\mathrm{F}}=0.6 \mathrm{~Hz}, 1 \mathrm{H}\right.$,
$\left.\mathrm{C}_{8} \mathrm{H}\right), 7.95\left(\mathrm{ddd},{ }^{3} \mathrm{~J}_{\mathrm{H}-\mathrm{H} 1}=8.4,{ }^{3} \mathrm{~J}_{\mathrm{H}-\mathrm{H} 4}=6.9,{ }^{4} \mathrm{~J}_{\mathrm{H}-\mathrm{H} 2}=1.3 \mathrm{~Hz}, 1 \mathrm{H}, \mathrm{C}_{6} \mathrm{H}\right)$, 7.83 (ddd, ${ }^{3} \mathrm{~J}_{\mathrm{H}-\mathrm{H} 2}=8.4,{ }^{3} \mathrm{~J}_{\mathrm{H}-\mathrm{H} 3}=6.9,{ }^{4} \mathrm{~J}_{\mathrm{H}-\mathrm{H} 1}=1.3 \mathrm{~Hz}, 1 \mathrm{H}, \mathrm{C}_{7} \mathrm{H}$ ), 7.13 $\left(\mathrm{t},{ }^{2} \mathrm{~J}_{\mathrm{H}-\mathrm{F}}=52.8 \mathrm{~Hz}, 1 \mathrm{H}, \mathrm{C}_{4} \mathrm{CHF}_{2}\right), 4.52\left(\mathrm{q},{ }^{3} \mathrm{~J}_{\mathrm{H}-\mathrm{H}}=7.2 \mathrm{~Hz}, 2 \mathrm{H}\right.$, $\left.\mathrm{C}_{3} \mathrm{OCH}_{2} \mathrm{CH}_{3}\right), 1.44\left(\mathrm{t},{ }^{3} \mathrm{JH}_{\mathrm{H}-\mathrm{H}}=7.2 \mathrm{~Hz}, 3 \mathrm{H}, \mathrm{C}_{3} \mathrm{OCH}_{2} \mathrm{CH}_{3}\right) \mathrm{ppm} .{ }^{19} \mathrm{~F}$ $\operatorname{NMR}\left(376 \mathrm{MHz}, \mathrm{CDCl}_{3}\right) \delta_{\mathrm{F}}=-64.17\left(\mathrm{~s}, \mathrm{C}_{2} \mathrm{CF}_{3}\right),-109.48\left(\mathrm{dd},{ }^{2} \mathrm{~J}_{F-H}\right.$ $\left.=52.7,{ }^{5} \mathrm{JF}_{-H}=2.0 \mathrm{~Hz}, \mathrm{C}_{4} \mathrm{CHF}_{2}\right) \mathrm{ppm} .{ }^{13} \mathrm{C} \mathrm{NMR}\left(101 \mathrm{MHz}, \mathrm{CDCl}_{3}\right)$ $\delta_{\mathrm{C}}=164.90\left(\mathrm{~s}, \mathrm{C}_{3} \mathrm{CO}\right), 147.30\left(\mathrm{~s}, \mathrm{C}_{10}\right), 143.72\left(\mathrm{q},{ }^{2} \mathrm{~J}_{\mathrm{C}-\mathrm{F}}=35.0 \mathrm{~Hz}\right.$, $\left.\mathrm{C}_{2}\right), 137.33\left(\mathrm{t},{ }^{2} \mathrm{~J}_{\mathrm{C}-\mathrm{F}}=23.5 \mathrm{~Hz}, \mathrm{C}_{4}\right), 132.19\left(\mathrm{~s}, \mathrm{C}_{6}\right), 131.17\left(\mathrm{~s}, \mathrm{C}_{8}\right)$, $130.75\left(\mathrm{~s}, \mathrm{C}_{7}\right), 125.37\left(\mathrm{t},{ }^{4} \mathrm{~J}_{\mathrm{C}-\mathrm{F}}=3.7 \mathrm{~Hz}, \mathrm{C}_{5}\right), 124.04\left(\mathrm{~s}, \mathrm{C}_{9}\right), 123.97$ (s, $\left.\mathrm{C}_{3}\right), 121.03$ (q, $\left.{ }^{1} \mathrm{~J}_{\mathrm{C}-\mathrm{F}}=276.7 \mathrm{~Hz}, \mathrm{C}_{2} \mathrm{CF}_{3}\right), 112.86\left(\mathrm{t},{ }^{1} \mathrm{~J}_{\mathrm{C}-\mathrm{F}}=241.8\right.$ $\mathrm{Hz}, \mathrm{C}_{4} \mathrm{CHF}_{2}$ ), 63.56 (s, $\mathrm{C}_{3} \mathrm{OCH}_{2} \mathrm{CH}_{3}$ ), 13.99 (s, $\left.\mathrm{C}_{3} \mathrm{OCH}_{2} \mathrm{CH}_{3}\right)$. IR $\mathrm{v}\left(\mathrm{cm}^{-1}\right): 2987-2901\left(\mathrm{C}_{\mathrm{sp} 3} \mathrm{H}\right), 1731\left(\mathrm{C}=\mathrm{O}_{\text {ester }}\right) . \mathrm{C}_{14} \mathrm{H}_{10} \mathrm{~F}_{5} \mathrm{NO}_{2}(319)$ : calcd (\%) N 4.39, C 52.67, H 3.16, found N 4.39, C 52.80, H 3.27. MP: $79.8-80.8^{\circ} \mathrm{C}$.

\section{Ethyl \\ 4-[fluoro(trifluoromethoxy)methyl]-2-} (trifluoromethyl)quinoline-3-carboxylate 5dD

The required FAR 1D was freshly prepared and activated by $\mathrm{BF}_{3} \bullet \mathrm{Et}_{2} \mathrm{O}$, in order to be used as the corresponding fluoroiminium salt 2D.

a) $\quad \mathrm{N}$-(1,2-Difluoro-2-(trifluoromethoxy)ethylidene)- $N$ methylmethanaminium tetrafluoroborate 2D (1.57 equiv., $852 \mathrm{mg}$, $3.06 \mathrm{mmol}$ ); b) Ethyl 4,4,4-trifluoro-3-(phenylamino)but-2-enoate 3d (1 equiv., $503 \mathrm{mg}, \quad 1.94 \mathrm{mmol}) ; \quad$ c) Ethyl 4[fluoro(trifluoromethoxy)methyl]-2-(trifluoromethyl)quinoline-3carboxylate $\mathbf{5 d D}$ was obtained after purification using a gradient of AcOEt in cyclohexane $(0-20 \%)$ as colourless solid in $74 \%$ yield (551 mg). ${ }^{1} \mathrm{H} \mathrm{NMR}\left(400 \mathrm{MHz}, \mathrm{CDCl}_{3}\right) \delta_{\mathrm{H}}=8.45\left(\mathrm{~d},{ }^{3} \mathrm{~J}_{\mathrm{H}-\mathrm{H}}=8.6 \mathrm{~Hz}\right.$, $\left.1 \mathrm{H}, \mathrm{C}_{5} \mathrm{H}\right), 8.31\left(\mathrm{dd},{ }^{3} \mathrm{H}_{\mathrm{H}-\mathrm{H}}=8.5,{ }^{6} \mathrm{~J}_{\mathrm{H}-\mathrm{F}}=0.6 \mathrm{~Hz}, 1 \mathrm{H}, \mathrm{C}_{8} \mathrm{H}\right), 7.95$ (ddd, $\left.{ }^{3} J_{H-H 1}=8.4,{ }^{3} J_{H-H 4}=6.9,{ }^{4} J_{H-H 2}=1.3 \mathrm{~Hz}, 1 \mathrm{H}, \mathrm{C}_{6} \mathrm{H}\right), 7.84\left(\mathrm{ddd},{ }^{3} \mathrm{~J}_{\mathrm{H}-}\right.$ $\left.H_{2}=8.4,{ }^{3} J_{H-H 3}=6.9,{ }^{4} J_{H-H 1}=1.3 \mathrm{~Hz}, 1 \mathrm{H}, \mathrm{C}_{7} \mathrm{H}\right), 7.21\left(\mathrm{~d},{ }^{2} \mathrm{~J}_{\mathrm{H}-\mathrm{F}}=\right.$ $\left.54.6 \mathrm{~Hz}, 1 \mathrm{H}, \mathrm{C}_{4} \mathrm{CHFOCF}_{3}\right), 4.62-4.43\left(\mathrm{~m}, 2 \mathrm{H}, \mathrm{C}_{3} \mathrm{OCH}_{2} \mathrm{CH}_{3}\right)$, $1.43\left(\mathrm{t},{ }^{3} \mathrm{~J}_{\mathrm{H}-\mathrm{H}}=7.2 \mathrm{~Hz}, 3 \mathrm{H}, \mathrm{C}_{3} \mathrm{OCH}_{2} \mathrm{CH}_{3}\right) \mathrm{ppm} .{ }^{19} \mathrm{~F} \mathrm{NMR}(376 \mathrm{MHz}$, $\left.\mathrm{CDCl}_{3}\right) \delta_{\mathrm{F}}=-59.40\left(\mathrm{~d},{ }^{4} J_{F-F}=4.8 \mathrm{~Hz}, \mathrm{C}_{4} \mathrm{CHFOCF}_{3}\right),-64.17(\mathrm{~s}$, $\left.\mathrm{C}_{2} C F_{3}\right),-120.38\left(\mathrm{dqd},{ }^{2} J_{F-H}=54.5,{ }^{4} J_{F-F}=4.9,{ }^{5} J_{F-H}=2.1 \mathrm{~Hz}\right.$, $\left.\mathrm{C}_{4} \mathrm{CHFOCF}_{3}\right) \mathrm{ppm} .{ }^{13} \mathrm{C} \mathrm{NMR}\left(101 \mathrm{MHz}, \mathrm{CDCl}_{3}\right) \delta_{\mathrm{c}}=164.87(\mathrm{~s}$, $\mathrm{C}_{3} \mathrm{CO}$ ), 147.43 (s, $\left.\mathrm{C}_{10}\right), 143.76\left(\mathrm{q},{ }^{2} \mathrm{~J}_{\mathrm{C}-\mathrm{F}}=35.2 \mathrm{~Hz}, \mathrm{C}_{2}\right), 136.59$ (d, $\left.{ }^{2} J_{C-F}=24.4 \mathrm{~Hz}, \mathrm{C}_{4}\right), 132.27\left(\mathrm{~s}, \mathrm{C}_{6}\right), 131.22\left(\mathrm{~s}, \mathrm{C}_{8}\right), 130.79\left(\mathrm{~s}, \mathrm{C}_{7}\right)$, $125.59\left(\mathrm{~d},{ }^{4} \mathrm{~J}_{\mathrm{C}-\mathrm{F}}=4.9 \mathrm{~Hz}, \mathrm{C}_{5}\right), 123.86\left(\mathrm{~s}, \mathrm{C}_{9}\right), 123.80\left(\mathrm{~s}, \mathrm{C}_{3}\right)$, 121.04 (qd, $\left.{ }^{1} \mathrm{~J}_{\mathrm{C}-F}=262.9,{ }^{3} \mathrm{~J}_{\mathrm{C}-\mathrm{F}}=1.6 \mathrm{~Hz}, \mathrm{C}_{4} \mathrm{CHFOCF}_{3}\right), 121.01$ (q, $\left.{ }^{1} J_{C-F}=276.7 \mathrm{~Hz}, C_{2} C F_{3}\right), 103.06\left(\mathrm{dq},{ }^{1} J_{C-F}=232.7,{ }^{3} J_{C-F}=3.9 \mathrm{~Hz}\right.$, $\mathrm{C}_{4} \mathrm{CHFOCF}_{3}$ ), 63.69 (s, $\left.\mathrm{C}_{3} \mathrm{OCH}_{2} \mathrm{CH}_{3}\right), 13.88\left(\mathrm{~s}, \mathrm{C}_{3} \mathrm{OCH}_{2} \mathrm{CH}_{3}\right)$ ppm. IR V $\left(\mathrm{cm}^{-1}\right): 2987-2901 \quad\left(\mathrm{C}_{\mathrm{sp} 3} \mathrm{H}\right), \quad 1728 \quad\left(\mathrm{C}=\mathrm{O}_{\text {ester }}\right)$. $\mathrm{C}_{15} \mathrm{H}_{10} \mathrm{~F}_{7} \mathrm{NO}_{3}$ (385): calcd (\%) N 3.64, C 46.77, H 2.62, found $\mathrm{N}$ 3.58, C 47.10, H 2.71. MP: $57.8-59.5^{\circ} \mathrm{C}$.

\section{POST-FUNCTIONALIZATION IN POSITION 3 OF QUINOLINE DERIVATIVES}

\section{Typical procedure $\boldsymbol{C}$ for the saponification reaction}

To a stirred solution of potassium hydroxide in ethanol/water 60:40 was added quinoline 5 . The reaction mixture was stirred under reflux for $4 \mathrm{~h}$, then quenched with concentrated hydrochloric acid until $\mathrm{pH}$ reached 1 . $\mathrm{EtOH}$ was removed under vacuum and organic compounds were extracted with AcOEt. The combined organic layers were washed with water, dried over $\mathrm{Na}_{2} \mathrm{SO}_{4}$, and concentrated under reduced pressure. 
The following experiments were carried out according to Typical Procedure $\mathbf{C}$, and specific details are reported as: a) $\mathrm{KOH}$ and ethanol/water mixture; b) quinoline 5 ; c) yield and aspect. Individual analysis for each compound (6) is given below. Atoms are numbered in the description of NMR spectra according to the Supporting Information.

\section{2,4-Bis(difluoromethyl)quinoline-3-carboxylic acid 6aA}

a) $\mathrm{KOH}$ (8 equiv., $10.6 \mathrm{~g}, 189 \mathrm{mmol}$ ), ethanol/water 60:40 (187 $\mathrm{ml}$ ); b) Ethyl 2,4-bis(difluoromethyl)quinoline-3-carboxylate 5aA (1 equiv., $7.14 \mathrm{~g}, 23.7 \mathrm{mmol}$ ); c) 2,4-Bis(difluoromethyl)quinoline3-carboxylic acid $6 \mathrm{aA}$ was obtained as a colourless solid in $99 \%$ yield $(6.44 \mathrm{~g}) .{ }^{1} \mathrm{H}$ NMR $(400 \mathrm{MHz}, \mathrm{DMSO}) \delta_{\mathrm{H}}=8.39\left(\mathrm{~d},{ }^{3} \mathrm{~J}_{\mathrm{H}-\mathrm{H}}=7.7\right.$ $\left.\mathrm{Hz}, 1 \mathrm{H}, \mathrm{C}_{5} \mathrm{H}\right), 8.27\left(\mathrm{~d},{ }^{3} \mathrm{~J}_{\mathrm{H}-\mathrm{H}}=7.8 \mathrm{~Hz}, 1 \mathrm{H}, \mathrm{C}_{8} \mathrm{H}\right), 8.07-7.99(\mathrm{~m}$, $\left.1 \mathrm{H}, \mathrm{C}_{6} \mathrm{H}\right), 7.97-7.88\left(\mathrm{~m}, 1 \mathrm{H}, \mathrm{C}_{7} \mathrm{H}\right), 7.70\left(\mathrm{t},{ }^{2} J_{\mathrm{H}-\mathrm{F}}=52.1 \mathrm{~Hz}, 1 \mathrm{H}\right.$, $\left.\mathrm{C}_{4} \mathrm{CHF}_{2}\right), 7.27\left(\mathrm{t},{ }^{2} \mathrm{~J}_{\mathrm{H}-\mathrm{F}}=53.7 \mathrm{~Hz}, 1 \mathrm{H}, \mathrm{C}_{2} \mathrm{CHF}_{2}\right) \mathrm{ppm} .{ }^{19} \mathrm{~F} \mathrm{NMR}(376$ $\mathrm{MHz}, \mathrm{DMSO}) \delta_{\mathrm{F}}=-106.25\left(\mathrm{dd},{ }^{2} \mathrm{~J}_{\mathrm{F}-H}=52.0,{ }^{5} J_{F-H}=1.7 \mathrm{~Hz}\right.$, $\left.\mathrm{C}_{4} \mathrm{CHF}_{2}\right),-110.60\left(\mathrm{~d},{ }^{2} J_{F-H}=53.7 \mathrm{~Hz}, \mathrm{C}_{2} \mathrm{CHF}_{2}\right) \mathrm{ppm} .{ }^{13} \mathrm{C} \mathrm{NMR}$ $(101 \mathrm{MHz}, \mathrm{DMSO}) \delta_{\mathrm{c}}=166.11\left(\mathrm{~s}, \mathrm{C}_{3} \mathrm{CO}\right), 147.73\left(\mathrm{t},{ }^{2} \mathrm{~J}_{\mathrm{C}-\mathrm{F}}=24.2\right.$ $\left.\mathrm{Hz}, \mathrm{C}_{2}\right), 146.40\left(\mathrm{~s}, \mathrm{C}_{10}\right), 135.53\left(\mathrm{t},{ }^{2} \mathrm{~J}_{\mathrm{C}-F}=22.7 \mathrm{~Hz}, \mathrm{C}_{4}\right), 132.02(\mathrm{~s}$, $\left.\mathrm{C}_{6}\right), 130.33\left(\mathrm{~s}, \mathrm{C}_{7}\right), 130.20\left(\mathrm{~s}, \mathrm{C}_{8}\right), 125.21\left(\mathrm{t},{ }^{3} \mathrm{~J}_{\mathrm{C}-\mathrm{F}}=6.1 \mathrm{~Hz}, \mathrm{C}_{3}\right)$, 124.74 (s, $\left.\mathrm{C}_{5}\right), 123.07$ (s, $\left.\mathrm{C}_{9}\right), 113.02\left(\mathrm{t},{ }^{1} \mathrm{~J}_{\mathrm{C}-\mathrm{F}}=241.7 \mathrm{~Hz}, \mathrm{C}_{2} \mathrm{CHF}_{2}\right.$ ), $112.87\left(\mathrm{t},{ }^{1} \mathrm{~J}_{\mathrm{C}-\mathrm{F}}=240.4 \mathrm{~Hz}, \mathrm{C}_{4} \mathrm{CHF}_{2}\right) \mathrm{ppm} . \mathrm{IR} \mathrm{v}\left(\mathrm{cm}^{-1}\right): 3418(\mathrm{OH})$, 1696 (C=Oacid). $\mathrm{C}_{12} \mathrm{H}_{7} \mathrm{~F}_{4} \mathrm{NO}_{2}$ (273): calcd (\%) N 5.13, C 52.76, $\mathrm{H}$ 2.58, found N 5.03, C 52.80, H 2.75. MP: $183.1-184{ }^{\circ} \mathrm{C}$.

\section{2-(Difluoromethyl)-4-}

[fluoro(trifluoromethoxy)methyl]quinoline-3-carboxylic acid 6aD

a) $\mathrm{KOH}$ (8 equiv., $305 \mathrm{mg}, 5.45 \mathrm{mmol}$ ), ethanol/water 60:40 (5.40 $\mathrm{ml}) ; \quad$ b) $\quad$ Ethyl 2-(difluoromethyl)-4[fluoro(trifluoromethoxy)methyl]quinoline-3-carboxylate $5 \mathrm{aD}$ (1 equiv., $250 \mathrm{mg}, \quad 0.681 \mathrm{mmol}$ ); c) 2-(Difluoromethyl)-4[fluoro(trifluoromethoxy)methyl]quinoline-3-carboxylic acid $\mathbf{6 a D}$ was obtained as a light beige solid in $86 \%$ yield $(197 \mathrm{mg}) .{ }^{1} \mathrm{H}$ NMR $(400 \mathrm{MHz}, \mathrm{DMSO}) \delta_{\mathrm{H}}=8.38\left(\mathrm{~d},{ }^{3} \mathrm{~J}_{\mathrm{H}-\mathrm{H}}=8.5 \mathrm{~Hz}, 1 \mathrm{H}, \mathrm{C}_{5} \mathrm{H}\right), 8.28(\mathrm{~d}$, $\left.{ }^{3} J_{H-H}=8.4 \mathrm{~Hz}, 1 \mathrm{H}, \mathrm{C}_{8} \mathrm{H}\right), 8.04\left(\mathrm{t},{ }^{3} \mathrm{~J}_{\mathrm{H}-\mathrm{H}}=7.7 \mathrm{~Hz}, 1 \mathrm{H}, \mathrm{C}_{6} \mathrm{H}\right), 7.96(\mathrm{t}$, $\left.{ }^{3} J_{H-H}=7.5 \mathrm{~Hz}, 1 \mathrm{H}, \mathrm{C}_{7} \mathrm{H}\right), 7.88\left(\mathrm{~d},{ }^{2} \mathrm{~J}_{\mathrm{H}-\mathrm{F}}=54.0 \mathrm{~Hz}, 1 \mathrm{H}\right.$, $\left.\mathrm{C}_{4} \mathrm{CHFOCF}_{3}\right), 7.30\left(\mathrm{t},{ }^{2} \mathrm{JH}_{\mathrm{H}-\mathrm{F}}=53.7 \mathrm{~Hz}, 1 \mathrm{H}, \mathrm{C}_{2} \mathrm{CHF}_{2}\right) \mathrm{ppm} .{ }^{19} \mathrm{~F} \mathrm{NMR}$ (376 MHz, DMSO) $\delta_{F}=-57.85\left(\mathrm{~d},{ }^{4} J_{F-F}=4.8 \mathrm{~Hz}, \mathrm{C}_{4} \mathrm{CHFOCF}_{3}\right)$, $114.57--116.64\left(\mathrm{~m}, \mathrm{~A}_{2} \mathrm{~B}_{2}, \Delta \mathrm{v}=459.45 \mathrm{~Hz}, \mathrm{C}_{2} \mathrm{CHF}_{2}\right),-122.18-$ $122.61\left(\mathrm{~m}, \mathrm{C}_{4} \mathrm{CHFOCF}_{3}\right) \mathrm{ppm} .{ }^{13} \mathrm{C} \mathrm{NMR}(101 \mathrm{MHz}, \mathrm{DMSO}) \delta_{\mathrm{C}}=$ $166.04\left(\mathrm{~s}, \mathrm{C}_{3} \mathrm{CO}\right), 147.96\left(\mathrm{t},{ }^{2} \mathrm{~J}_{\mathrm{C}-F}=23.8 \mathrm{~Hz}, \mathrm{C}_{2}\right), 146.51$ (s, $\left.\mathrm{C}_{9}\right)$, $134.67\left(\mathrm{~d},{ }^{2} \mathrm{~J}_{\mathrm{C}-\mathrm{F}}=23.3 \mathrm{~Hz}, \mathrm{C}_{4}\right), 132.12\left(\mathrm{~s}, \mathrm{C}_{7}\right), 130.43\left(\mathrm{~s}, \mathrm{C}_{6}\right)$, $130.34\left(\mathrm{~s}, \mathrm{C}_{8}\right), 125.59-125.22\left(\mathrm{~m}, \mathrm{C}_{3}\right), 124.71\left(\mathrm{~d},{ }^{4} \mathrm{~J}_{\mathrm{C}-\mathrm{F}}=3.9 \mathrm{~Hz}\right.$, $\left.\mathrm{C}_{5}\right), 122.86\left(\mathrm{~s}, \mathrm{C}_{10}\right), 120.55$ (qd, ${ }^{1} \mathrm{~J}_{\mathrm{C}-F}=261.6,{ }^{3} \mathrm{~J}_{\mathrm{C}-F}=1.2 \mathrm{~Hz}$, $\left.\mathrm{C}_{4} \mathrm{CHFOCF}_{3}\right), 112.80\left(\mathrm{t},{ }^{1} \mathrm{JC}_{\mathrm{C}-\mathrm{F}}=241.5 \mathrm{~Hz}, \mathrm{C}_{2} \mathrm{CHF}_{2}\right), 103.06(\mathrm{dq}$ $\left.{ }^{1} J_{C-F}=230.2,{ }^{3} J_{C-F}=3.8 \mathrm{~Hz}, C_{4} C H F O C F_{3}\right)$ ppm. IR v $\left(\mathrm{cm}^{-1}\right): 2920$ $\left(\mathrm{C}_{\mathrm{sp} 3} \mathrm{H}\right), 1714\left(\mathrm{C}=\mathrm{O}_{\text {acid }}\right) . \mathrm{C}_{13} \mathrm{H}_{7} \mathrm{~F}_{6} \mathrm{NO}_{3}$ (339): calcd (\%) N 4.13, C 46.03, H 2.08, found N 4.02, C 46.87, H 2.39. MP: $183.3-$ $185.5^{\circ} \mathrm{C}$.

6-Chloro-2,4-bis(difluoromethyl)quinoline-3-carboxylic acid 6cA

a) $\mathrm{KOH}$ (8 equiv., $0.585 \mathrm{~g}, 10.4 \mathrm{mmol}$ ), ethanol/water $60: 40$ (10.30 $\mathrm{ml}$; b) Ethyl 6-chloro-2,4-bis(difluoromethyl)quinoline-3carboxylate $5 \mathrm{cA}$ (1 equiv., $0.583 \mathrm{~g}, 1.3 \mathrm{mmol}$ ); c) 6-Chloro-2,4- bis(difluoromethyl)quinoline-3-carboxylic acid 6cA was obtained as a light beige solid in $41 \%$ yield $(531 \mathrm{mg}) .{ }^{1} \mathrm{H}$ NMR $(400 \mathrm{MHz}$, Acetone $\left.{ }^{\mathrm{d} 6}\right) \delta_{\mathrm{H}}=8.45\left(\mathrm{dd},{ }^{4} \mathrm{~J}_{\mathrm{H}-\mathrm{H}}=3.7,{ }^{5} \mathrm{~J}_{\mathrm{H}-\mathrm{F}}=1.8 \mathrm{~Hz}, 1 \mathrm{H}, \mathrm{C}_{5} \mathrm{H}\right)$, $8.30\left(\mathrm{~d},{ }^{3} \mathrm{~J}_{\mathrm{H}-\mathrm{H}}=9.0 \mathrm{~Hz}, 1 \mathrm{H}, \mathrm{C} 8 \mathrm{H}\right), 8.04\left(\mathrm{dd},{ }^{3} \mathrm{~J}_{\mathrm{H}-\mathrm{H}}=9.0,{ }^{4} \mathrm{~J}_{\mathrm{H}-\mathrm{H}}=2.2\right.$ $\mathrm{Hz}, 1 \mathrm{H}, \mathrm{C}_{7} \mathrm{H}$ ), 7.63 (t, ${ }^{2} J_{H-F}=52.3 \mathrm{~Hz}, 1 \mathrm{H}, \mathrm{C}_{4} \mathrm{CHF}_{2}$ ), 7.20 (t, ${ }^{2} \mathrm{~J}_{\mathrm{H}-\mathrm{F}}$ $\left.=54.1 \mathrm{~Hz}, 1 \mathrm{H}, \mathrm{C}_{2} \mathrm{CHF}_{2}\right) \mathrm{ppm} .{ }^{19} \mathrm{~F} \mathrm{NMR}\left(376 \mathrm{MHz}\right.$, Acetone $\left.{ }^{\mathrm{d} 6}\right) \delta_{\mathrm{F}}$ $=-111.47\left(\mathrm{~d},{ }^{2} J_{F-H}=52.2 \mathrm{~Hz}, \mathrm{C}_{4} C H F_{2}\right),-116.87\left(\mathrm{~d},{ }^{2} J_{F-H}=54.1 \mathrm{~Hz}\right.$, $\left.\mathrm{C}_{2} \mathrm{CHF}_{2}\right)$ ppm. ${ }^{13} \mathrm{C}$ NMR $\left(101 \mathrm{MHz}\right.$, Acetone $\left.{ }^{\mathrm{d} 6}\right) \delta_{\mathrm{c}}=166.01(\mathrm{~s}$, $\mathrm{C}_{3} \mathrm{CO}$ ), 149.66 (t, $\left.{ }^{2} \mathrm{~J}_{\mathrm{C}-\mathrm{F}}=24.7 \mathrm{~Hz}, \mathrm{C}_{2}\right), 146.77$ (s, $\left.\mathrm{C}_{6}\right), 136.89$ (t, $\left.{ }^{2} J_{C-F}=23.4 \mathrm{~Hz}, \mathrm{C}_{4}\right), 136.61\left(\mathrm{~s}, \mathrm{C}_{10}\right), 133.57\left(\mathrm{~s}, \mathrm{C}_{7}\right), 133.43\left(\mathrm{~s}, \mathrm{C}_{8}\right)$, $126.53\left(\mathrm{t},{ }^{3} \mathrm{~J}_{\mathrm{C}-\mathrm{F}}=6.4 \mathrm{~Hz}, \mathrm{C}_{3}\right), 125.03\left(\mathrm{~s}, \mathrm{C}_{9}\right), 124.94\left(\mathrm{t},{ }^{4} \mathrm{~J}_{\mathrm{C}-F}=3.7\right.$ $\left.\mathrm{Hz}, \mathrm{C}_{5}\right), 114.20\left(\mathrm{t},{ }^{1} \mathrm{~J}_{\mathrm{C}-\mathrm{F}}=242.1 \mathrm{~Hz}, \mathrm{C}_{2} \mathrm{CHF}_{2}\right), 114.05$ (t, ${ }^{1} \mathrm{~J}_{\mathrm{C}-\mathrm{F}}=$ $\left.240.4 \mathrm{~Hz}, \mathrm{C}_{4} \mathrm{CHF}_{2}\right) \mathrm{ppm}$. IR v $\left(\mathrm{cm}^{-1}\right): 2918\left(\mathrm{C}_{\mathrm{sp} 3} \mathrm{H}\right), 1725-1706$ (C=Oacid). $\mathrm{C}_{12} \mathrm{H}_{6} \mathrm{~F}_{4} \mathrm{NClO}_{2}$ (307): calcd (\%) N 4.55, C 46.85, H 1.97, found N 4.43, C 47.22, H 2.27. MP: $236.2-237.2^{\circ} \mathrm{C}$.

Carbamate synthesis: $\quad t$-Butyl $\quad N$-[2,4-
bis(difluoromethyl)quinolin-3-yl]carbamate 7aA

To a solution of 2,4-bis(difluoromethyl)quinoline-3-carboxylic acid 6aA (1 equiv., $802 \mathrm{mg}, 2.56 \mathrm{mmol}$ ) in tert-butyl alcohol $(10 \mathrm{~mL}$ ) were added $\mathrm{NEt}_{3}$ (1.6 equiv., $0.552 \mathrm{~mL}, 4.09 \mathrm{mmol}$ ) and DPPA (1.3 equiv., $0.718 \mathrm{~mL}, 3.32 \mathrm{mmol}$ ). The reactor was not totally sealed in order to allow gas evacuation and the mixture was stirred at $100^{\circ} \mathrm{C}$ overnight. The reaction mixture was cooled down and diluted with water. The organic layer was extracted with AcOEt, washed with a solution of $\mathrm{NaHCO}_{3}$ followed by brine, dried over $\mathrm{Na}_{2} \mathrm{SO}_{4}$ and concentrated under vacuum. The product was purified by flash chromatography using a gradient of AcOEt in cyclohexane (0-20\%). $t$-Butyl $N$-[2,4-bis(difluoromethyl)quinolin3 -yl]carbamate $7 \mathrm{aA}$ was obtained as a beige solid in $64 \%$ yield $(0.567 \mathrm{~g}) .{ }^{1} \mathrm{H}$ NMR $\left(400 \mathrm{MHz}, \mathrm{CDCl}_{3}\right) \delta_{\mathrm{H}}=8.47\left(\mathrm{~d},{ }^{3} \mathrm{~J}_{\mathrm{H}-\mathrm{H}}=7.9 \mathrm{~Hz}\right.$, $\left.1 \mathrm{H}, \mathrm{C}_{5} \mathrm{H}\right), 8.16\left(\mathrm{~d},{ }^{3} \mathrm{~J}_{\mathrm{H}-\mathrm{H}}=8.5 \mathrm{~Hz}, 1 \mathrm{H}, \mathrm{C}_{8} \mathrm{H}\right), 7.82\left(\mathrm{t},{ }^{3} \mathrm{~J}_{\mathrm{H}-\mathrm{H}}=7.4 \mathrm{~Hz}\right.$, $\left.1 \mathrm{H}, \mathrm{C}_{7} \mathrm{H}\right), 7.73\left(\mathrm{t},{ }^{3} \mathrm{~J}_{\mathrm{H}-\mathrm{H}}=7.5 \mathrm{~Hz}, 1 \mathrm{H}, \mathrm{C}_{6} \mathrm{H}\right), 7.21\left(\mathrm{t},{ }^{2} \mathrm{~J}_{\mathrm{H}-\mathrm{F}}=53.7 \mathrm{~Hz}\right.$, $\left.1 \mathrm{H}, \mathrm{C}_{4} \mathrm{CHF}_{2}\right), 6.85\left(\mathrm{t},{ }^{2} \mathrm{~J}_{\mathrm{H}-\mathrm{F}}=54.2 \mathrm{~Hz}, 1 \mathrm{H}, \mathrm{C}_{2} \mathrm{CHF}_{2}\right), 6.65(\mathrm{~s}, 1 \mathrm{H}$, $\mathrm{NH}), 1.69-1.20\left(\mathrm{~m}, 9 \mathrm{H}, t\right.$-Bu) ppm. ${ }^{19} \mathrm{~F}$ NMR $\left(376 \mathrm{MHz}, \mathrm{CDCl}_{3}\right)$ $\delta_{F}=-112.09\left(\mathrm{~s}, \mathrm{C}_{4} \mathrm{CHF}_{2}\right),-113.57\left(\mathrm{~d},{ }^{2} \mathrm{~J}_{F-H}=52.5 \mathrm{~Hz}, \mathrm{C}_{2} \mathrm{CHF}_{2}\right)$ ppm. ${ }^{13} \mathrm{C}$ NMR $\left(101 \mathrm{MHz}, \mathrm{CDCl}_{3}\right) \delta_{\mathrm{C}}=154.31\left(\mathrm{~s}, \mathrm{C}_{11}\right), 149.51-$ $146.66\left(\mathrm{~m}, \mathrm{C}_{2}\right), 146.01\left(\mathrm{~s}, \mathrm{C}_{10}\right), 137.39-134.67\left(\mathrm{~m}, \mathrm{C}_{4}\right), 130.46$ $\left(\mathrm{s}, \mathrm{C}_{7,8}\right), 129.61\left(\mathrm{~s}, \mathrm{C}_{6}\right), 127.05\left(\mathrm{~s}, \mathrm{C}_{9}\right), 125.59\left(\mathrm{t},{ }^{4} \mathrm{~J}_{\mathrm{C}-\mathrm{F}}=3.9 \mathrm{~Hz}\right.$, $\left.\mathrm{C}_{5}\right), 125.13\left(\mathrm{~s}, \mathrm{C}_{3}\right), 116.89\left(\mathrm{t},{ }^{1} \mathrm{~J}_{\mathrm{C}-\mathrm{F}}=240.1 \mathrm{~Hz}, \mathrm{C}_{2} \mathrm{CHF}_{2}\right), 112.55$ $\left(\mathrm{t},{ }^{1} \mathrm{~J}_{\mathrm{C}-\mathrm{F}}=238.5 \mathrm{~Hz}, \mathrm{C}_{4} \mathrm{CHF}_{2}\right), 82.57$ (s, $\left.\mathrm{C}_{12}\right), 28.14$ (s, $\left.t-\mathrm{Bu}\right) \mathrm{ppm}$. IR $\vee\left(\mathrm{cm}^{-1}\right): 3675(\mathrm{NH}), 2978-2901\left(\mathrm{C}_{\mathrm{sp} 3} \mathrm{H}\right), 1704\left(\mathrm{C}=\mathrm{O}_{\text {ester }}\right)$. $\mathrm{C}_{16} \mathrm{H}_{16} \mathrm{~F}_{4} \mathrm{~N}_{2} \mathrm{O}_{2}$ (344): calcd (\%) N 8.14, C 55.81, H 4.68, found $\mathrm{N}$ 8.01, C 56.22, H 4.79. MP: $135.6-136.5^{\circ} \mathrm{C}$.

\section{2,4-Bis(difluoromethyl)quinolin-3-amine 8aA}

$t$-Butyl $N$-[2,4-bis(difluoromethyl)quinolin-3-yl]carbamate 7aA (1 equiv., $566 \mathrm{mg}, 1.32 \mathrm{mmol}$ ) was dissolved in DCE (4.53 mL) and treated with TFA (20.9 equiv., $2.04 \mathrm{~mL}, 27.5 \mathrm{mmol}$ ). After being stirred for $4 \mathrm{~h}$ at room temperature, the mixture was quenched with water and a solution of $\mathrm{NaHCO}_{3}$ until neutral $\mathrm{pH}$ was reached. The organic layer was extracted with DCM, washed with water, dried over $\mathrm{Na}_{2} \mathrm{SO}_{4}$ and concentrated under reduced pressure. 2,4-Bis(difluoromethyl)quinolin-3-amine $\mathbf{8 a A}$ was obtained as a yellow solid in $98 \%$ yield $(315 \mathrm{mg}) .{ }^{1} \mathrm{H}$ NMR $\left(400 \mathrm{MHz}, \mathrm{CDCl}_{3}\right) \delta_{\mathrm{H}}$ $=8.00\left(\mathrm{dd},{ }^{3} \mathrm{~J}_{\mathrm{H}-\mathrm{H}}=8.3,{ }^{4} \mathrm{~J}_{\mathrm{H}-\mathrm{H}}=1.1 \mathrm{~Hz}, 1 \mathrm{H}, \mathrm{C}_{5} \mathrm{H}\right), 7.83\left(\mathrm{~d},{ }^{3} \mathrm{~J}_{\mathrm{H}-\mathrm{H}}=\right.$ $\left.8.6 \mathrm{~Hz}, 1 \mathrm{H}, \mathrm{C}_{8} \mathrm{H}\right), 7.62-7.57\left(\mathrm{~m}, 1 \mathrm{H}, \mathrm{C}_{6} \mathrm{H}\right), 7.51$ (ddd, ${ }^{3} \mathrm{~J}_{\mathrm{H}-\mathrm{H} 2}=8.2$, 
$\left.{ }^{3} J_{H-H 3}=6.9,{ }^{4} J_{H-H 1}=1.3 \mathrm{~Hz}, 1 \mathrm{H}, \mathrm{C}_{7} \mathrm{H}\right), 7.43\left(\mathrm{t},{ }^{2} J_{H-F}=53.7 \mathrm{~Hz}, 1 \mathrm{H}\right.$, $\left.\mathrm{C}_{4} \mathrm{CHF}_{2}\right), 6.84\left(\mathrm{t},{ }^{2} \mathrm{~J}_{\mathrm{H}-\mathrm{F}}=54.2 \mathrm{~Hz}, 1 \mathrm{H}, \mathrm{C}_{2} \mathrm{CHF}_{2}\right), 5.08\left(\mathrm{~s}, 2 \mathrm{H}, \mathrm{NH}_{2}\right)$ ppm. ${ }^{19} \mathrm{~F}$ NMR $\left(376 \mathrm{MHz}, \mathrm{CDCl}_{3}\right) \delta_{\mathrm{F}}=-116.50\left(\mathrm{~d},{ }^{2} \mathrm{~J}_{\mathrm{F}-\mathrm{H}}=56.4 \mathrm{~Hz}\right.$, $\left.\mathrm{C}_{4} \mathrm{CHF}_{2}\right),-116.63\left(\mathrm{~d},{ }^{2} \mathrm{~J}_{\mathrm{F}-\mathrm{H}}=52.6 \mathrm{~Hz}, \mathrm{C}_{2} \mathrm{CHF}_{2}\right) \mathrm{ppm} .{ }^{13} \mathrm{C} \mathrm{NMR}(101$ $\left.\mathrm{MHz}, \mathrm{CDCl}_{3}\right) \delta_{\mathrm{C}}=141.95\left(\mathrm{t},{ }^{2} \mathrm{~J}_{\mathrm{C}-\mathrm{F}}=26.1 \mathrm{~Hz}, \mathrm{C}_{2}\right), 140.35\left(\mathrm{~s}, \mathrm{C}_{9}\right)$, $137.10\left(\mathrm{~s}, \mathrm{C}_{3}\right), 130.85\left(\mathrm{~s}, \mathrm{C}_{5}\right), 129.93\left(\mathrm{~s}, \mathrm{C}_{6}\right), 126.92\left(\mathrm{t},{ }^{3} \mathrm{~J}_{\mathrm{C}-\mathrm{F}}=4.9\right.$ $\left.\mathrm{Hz}, \mathrm{C}_{10}\right), 126.21$ (s, $\left.\mathrm{C}_{7}\right), 119.97\left(\mathrm{~s}, \mathrm{C}_{8}\right), 118.65\left(\mathrm{t},{ }^{1} \mathrm{~J}_{\mathrm{C}-F}=239.4 \mathrm{~Hz}\right.$, $\left.\mathrm{C}_{2} \mathrm{CHF}_{2}\right), 114.58\left(\mathrm{t},{ }^{2} \mathrm{~J}_{\mathrm{C}-\mathrm{F}}=20.4 \mathrm{~Hz}, \mathrm{C}_{4}\right), 113.90\left(\mathrm{t},{ }^{1} \mathrm{~J}_{\mathrm{C}-\mathrm{F}}=234.9\right.$ $\left.\mathrm{Hz}, \mathrm{C}_{4} \mathrm{CHF}_{2}\right)$ ppm. IR v $\left(\mathrm{cm}^{-1}\right): 3533-3238\left(\mathrm{NH}+\mathrm{C}_{\mathrm{sp} 2 \mathrm{H}}\right), 1635-$ $1591(\mathrm{C}=\mathrm{C}) . \mathrm{C}_{11} \mathrm{H}_{8} \mathrm{~F}_{4} \mathrm{~N}_{2}(244)$ : calcd (\%) N 11.37, C 54.11, H 3.30, found N 11.34, C 54.19, H 3.39. MP: $68.3-70.3^{\circ} \mathrm{C}$.

\section{Typical procedure $D$ for the Sandmeyer reaction}

To a solution of 2,4-bis(difluoromethyl)quinolin-3-amine 8aA (1 equiv.) in dry MeCN under argon was added the copper precursor (1 equiv.). The suspension was stirred for $10 \mathrm{~min}$ and tert-butyl nitrite (4 equiv.) was added. The mixture was stirred for $20 \mathrm{~min}$ at room temperature. Then the flask was fitted with a reflux condenser and heated at $60{ }^{\circ} \mathrm{C}$ for $4 \mathrm{~h}$. After cooling to room temperature, the mixture was diluted with saturated aqueous $\mathrm{NaHCO}_{3}$ and extracted with AcOEt. The organic layer was washed with water and brine, dried over $\mathrm{Na}_{2} \mathrm{SO}_{4}$ and concentrated under reduced pressure.

The following experiments were carried out according to Typical Procedure $D$, and specific details are reported as: a) Amine; b) $\mathrm{MeCN}$; c) Copper precursor; d) $t$-BuONO; and e) yield and aspect. Individual analysis for each compound (9aA, 10aA and 11aA) is given below. Atoms are numbered in the description of NMR spectra according to the Supporting Information.

\section{2,4-Bis(difluoromethyl)-3-iodoquinoline 9aA}

a) 2,4-Bis(difluoromethyl)quinolin-3-amine $8 \mathrm{aA}$ (1 equiv., $231 \mathrm{mg}$, $0.475 \mathrm{mmol}$ ); b) MeCN (4.97 mL); c) Copper (I) iodide (1 equiv., $90.4 \mathrm{mg}, 0.475 \mathrm{mmol}$ ); d) $t$-BuONO (4 equiv., $0.228 \mathrm{~mL}, 1.9$ mmol); e) 2,4-Bis(difluoromethyl)-3-iodoquinoline $9 \mathrm{aA}$ was obtained as a colourless solid after purification on flash chromatography using a gradient of AcOEt in cyclohexane (0$20 \%)$ in $65 \%$ yield $(109 \mathrm{mg}) .{ }^{1} \mathrm{H} \mathrm{NMR}\left(400 \mathrm{MHz}, \mathrm{CDCl}_{3}\right) \delta_{\mathrm{H}}=8.44$ $\left(\mathrm{d},{ }^{3} \mathrm{~J}_{\mathrm{H}-\mathrm{H}}=8.1 \mathrm{~Hz}, 1 \mathrm{H}, \mathrm{C}_{5} \mathrm{H}\right), 8.23\left(\mathrm{~d},{ }^{3} \mathrm{~J}_{\mathrm{H}-\mathrm{H}}=8.4 \mathrm{~Hz}, 1 \mathrm{H}, \mathrm{C}_{8} \mathrm{H}\right), 7.87$ (ddd, ${ }^{3} \mathrm{~J}_{\mathrm{H}-\mathrm{H} 1}=8.4,{ }^{3} \mathrm{~J}_{\mathrm{H}-\mathrm{H} 4}=6.9,{ }^{4} \mathrm{~J}_{\mathrm{H}-\mathrm{H} 2}=1.3 \mathrm{~Hz}, 1 \mathrm{H}, \mathrm{C}_{6} \mathrm{H}$ ), 7.75 (ddd $\left.{ }^{3} J_{H-H 2}=8.4,{ }^{3} J_{H-H 3}=6.9,{ }^{4} J_{H-H 1}=1.3 \mathrm{~Hz}, 1 \mathrm{H}, \mathrm{C} 7 \mathrm{H}\right), 7.51\left(\mathrm{t},{ }^{2} J_{H-F}=\right.$ $\left.52.7 \mathrm{~Hz}, 1 \mathrm{H}, \mathrm{C}_{4} \mathrm{CHF}_{2}\right), 7.06\left(\mathrm{t},{ }^{2} \mathrm{~J}_{\mathrm{H}-\mathrm{F}}=54.0 \mathrm{~Hz}, 1 \mathrm{H}, \mathrm{C}_{2} \mathrm{CHF}_{2}\right) \mathrm{ppm}$. ${ }^{19} \mathrm{~F} \mathrm{NMR}\left(376 \mathrm{MHz}, \mathrm{CDCl}_{3}\right) \delta_{\mathrm{F}}=-109.46\left(\mathrm{dd},{ }^{2} \mathrm{~J}_{\mathrm{F}-\mathrm{H}}=52.7,{ }^{5} \mathrm{~J}_{\mathrm{F}-\mathrm{H}}=\right.$ $\left.2.4 \mathrm{~Hz}, \mathrm{C}_{4} \mathrm{CHF}_{2}\right),-115.42\left(\mathrm{~d},{ }^{2} J_{F-H}=54.0 \mathrm{~Hz}, \mathrm{C}_{2} \mathrm{CHF}_{2}\right) \mathrm{ppm} .{ }^{13} \mathrm{C}$ $\operatorname{NMR}\left(101 \mathrm{MHz}, \mathrm{CDCl}_{3}\right) \delta_{\mathrm{c}}=150.58\left(\mathrm{t},{ }^{2} J_{\mathrm{C}-\mathrm{F}}=23.4 \mathrm{~Hz}, \mathrm{C}_{2}\right)$, $147.13\left(\mathrm{~s}, \mathrm{C}_{10}\right), 141.31\left(\mathrm{t},{ }^{2} \mathrm{~J}_{\mathrm{C}-\mathrm{F}}=24.0 \mathrm{~Hz}, \mathrm{C}_{4}\right), 131.38\left(\mathrm{~s}, \mathrm{C}_{6}\right)$, 130.66 (s, C 8 ), 129.87 (s, $\left.\mathrm{C}_{7}\right), 126.04\left(\mathrm{~s}, \mathrm{C}_{9}\right), 124.51\left(\mathrm{t},{ }^{4} \mathrm{~J}_{\mathrm{C}-\mathrm{F}}=5.2\right.$ $\left.\mathrm{Hz}, \mathrm{C}_{5}\right), 121.69\left(\mathrm{t},{ }^{1} \mathrm{~J}_{\mathrm{C}-\mathrm{F}}=242.4 \mathrm{~Hz}, \mathrm{C}_{4} \mathrm{CHF}_{2}\right), 115.50\left(\mathrm{t},{ }^{1} \mathrm{~J}_{\mathrm{C}-\mathrm{F}}=\right.$ $244.5 \mathrm{~Hz}, \mathrm{C}_{2} \mathrm{CHF}_{2}$ ), 89.31 (t, ${ }^{3} \mathrm{~J}_{\mathrm{C}-\mathrm{F}}=8.1 \mathrm{~Hz}, \mathrm{C}_{3}$ ) ppm. $\mathrm{C}_{11} \mathrm{H}_{6} \mathrm{IF}_{4} \mathrm{~N}$ (355): calcd (\%) N 3.94, C 37.21, H 1.70, found N 3.96, C 37.33, H 1.78. MP: $87.1-87.9^{\circ} \mathrm{C}$.

\section{3-Bromo-2,4-bis(difluoromethyl)quinoline 10aA}

a) 2,4-Bis(difluoromethyl)quinolin-3-amine $8 \mathrm{aA}$ (1 equiv., $245 \mathrm{mg}$, $1 \mathrm{mmol}$ ); b) $\mathrm{MeCN}(10.5 \mathrm{~mL})$; c) Copper (II) bromide (0.5 equiv., $112 \mathrm{mg}, 0.502 \mathrm{mmol}$ ); d) $t$-BuONO (4 equiv., $0.481 \mathrm{~mL}, 4.01$ $\mathrm{mmol}$ ); e) 3-Bromo-2,4-bis(difluoromethyl)quinoline 10aA was obtained as a colourless solid after purification on flash chromatography using a gradient of AcOEt in cyclohexane (0-5\%) in $50 \%$ yield $(153 \mathrm{mg}) .{ }^{1} \mathrm{H}$ NMR $\left(400 \mathrm{MHz}, \mathrm{CDCl}_{3}\right) \delta_{\mathrm{H}}=8.45$ (dd, $\left.{ }^{3} J_{H-H}=8.6,{ }^{5} J_{H-F}=2.0 \mathrm{~Hz}, 1 \mathrm{H}, \mathrm{C}_{5} \mathrm{H}\right), 8.24\left(\mathrm{~d},{ }^{3} \mathrm{~J}_{\mathrm{H}-\mathrm{H}}=8.2 \mathrm{~Hz}, 1 \mathrm{H}\right.$, $\left.\mathrm{C}_{8} \mathrm{H}\right), 7.87\left(\mathrm{ddd},{ }^{3} \mathrm{~J}_{\mathrm{H}-\mathrm{H} 2}=8.4,{ }^{3} \mathrm{~J}_{\mathrm{H}-\mathrm{H} 4}=6.9,{ }^{4} \mathrm{~J}_{\mathrm{H}-\mathrm{H} 1}=1.3 \mathrm{~Hz}, 1 \mathrm{H}, \mathrm{C}_{7} \mathrm{H}\right)$, 7.77 (ddd, $\left.{ }^{3} J_{H-H 1}=8.5,{ }^{3} J_{H-H 3}=6.9,{ }^{4} J_{H-H 2}=1.3 \mathrm{~Hz}, 1 \mathrm{H}, \mathrm{C}_{6} \mathrm{H}\right), 7.59$ $\left(\mathrm{t},{ }^{2} J_{H-F}=52.9 \mathrm{~Hz}, 1 \mathrm{H}, \mathrm{C}_{4} \mathrm{CHF}_{2}\right), 7.06\left(\mathrm{t},{ }^{2} J_{\mathrm{H}-\mathrm{F}}=53.8 \mathrm{~Hz}, 1 \mathrm{H}\right.$, $\left.\mathrm{C}_{2} \mathrm{CHF}_{2}\right) \mathrm{ppm} .{ }^{19} \mathrm{~F} \mathrm{NMR}\left(376 \mathrm{MHz}, \mathrm{CDCl}_{3}\right) \delta_{\mathrm{F}}=-111.19\left(\mathrm{dd},{ }^{2} J_{\mathrm{F}-\mathrm{H}}\right.$ $\left.=52.9,{ }^{5} J_{F-H}=2.3 \mathrm{~Hz}, \mathrm{C}_{4} C H F_{2}\right),-116.95\left(\mathrm{~d},{ }^{2} J_{F-H}=53.8 \mathrm{~Hz}\right.$, $\left.\mathrm{C}_{2} \mathrm{CHF}_{2}\right) \mathrm{ppm} .{ }^{13} \mathrm{C}$ NMR $\left(101 \mathrm{MHz}, \mathrm{CDCl}_{3}\right) \delta_{\mathrm{C}}=148.80\left(\mathrm{t},{ }^{2} \mathrm{~J}_{\mathrm{C}-\mathrm{F}}=\right.$ $\left.23.7 \mathrm{~Hz}, \mathrm{C}_{2}\right), 146.53\left(\mathrm{~s}, \mathrm{C}_{10}\right), 137.87\left(\mathrm{t},{ }^{2} J_{\mathrm{C}-F}=24.4 \mathrm{~Hz}, \mathrm{C}_{4}\right)$, $131.20\left(\mathrm{~s}, \mathrm{C}_{7}\right), 130.90\left(\mathrm{~s}, \mathrm{C}_{8}\right), 130.19$ (s, $\left.\mathrm{C}_{6}\right), 125.97$ (s, $\left.\mathrm{C}_{9}\right)$, $124.78\left(\mathrm{t},{ }^{4} \mathrm{~J}_{\mathrm{C}-\mathrm{F}}=4.8 \mathrm{~Hz}, \mathrm{C}_{5}\right), 115.80\left(\mathrm{t},{ }^{1} \mathrm{~J}_{\mathrm{C}-\mathrm{F}}=193.9 \mathrm{~Hz}, \mathrm{C}_{4} \mathrm{CHF}_{2}\right)$, $114.95\left(\mathrm{t},{ }^{3} \mathrm{~J}_{\mathrm{C}-\mathrm{F}}=7.7 \mathrm{~Hz}, \mathrm{C}_{3}\right), 113.88\left(\mathrm{t},{ }^{1} \mathrm{JC}_{\mathrm{C}-\mathrm{F}}=196.9 \mathrm{~Hz}, \mathrm{C}_{2} \mathrm{CHF}_{2}\right.$ ) ppm. $\mathrm{C}_{11} \mathrm{H}_{6} \mathrm{BrF} 4 \mathrm{~N}$ (308): calcd (\%) N 4.55, C 42.89, H 1.96, found $\mathrm{N} 4.47, \mathrm{C} 43.05, \mathrm{H} 2.13$. MP: $68.8-70^{\circ} \mathrm{C}$.

\section{2,4-Bis(difluoromethyl)quinoline-3-carbonitrile 11aA}

a) 2,4-Bis(difluoromethyl)quinolin-3-amine 8aA (1 equiv., $247 \mathrm{mg}$, $1.01 \mathrm{mmol}$ ); b) MeCN (5 mL); c) Copper (I) cyanide (1 equiv., 90.6 $\mathrm{mg}, 1.01 \mathrm{mmol}$ ); d) $t$-BuONO (4 equiv., $0.485 \mathrm{~mL}, 4.05 \mathrm{mmol}$ ); e) 2,4-Bis(difluoromethyl)quinoline-3-carbonitrile 11aA was obtained as a colourless solid after purification on flash chromatography using a gradient of AcOEt in cyclohexane (0-5\%) in $47 \%$ yield $(121 \mathrm{mg}) .{ }^{1} \mathrm{H} \mathrm{NMR}\left(400 \mathrm{MHz}, \mathrm{CDCl}_{3}\right) \delta_{\mathrm{H}}=8.51$ (dd, $\left.{ }^{3} J_{H-H}=8.6,{ }^{5} J_{H-F}=1.7 \mathrm{~Hz}, 1 \mathrm{H}, \mathrm{C}_{5} \mathrm{H}\right), 8.30\left(\mathrm{~d},{ }^{3} J_{\mathrm{H}-\mathrm{H}}=8.4 \mathrm{~Hz}, 1 \mathrm{H}\right.$, $\left.\mathrm{C}_{8} \mathrm{H}\right), 8.04$ (ddd, ${ }^{3} \mathrm{~J}_{\mathrm{H}-\mathrm{H} 2}=8.4,{ }^{3} \mathrm{~J}_{\mathrm{H}-\mathrm{H} 4}=7.0,{ }^{4} \mathrm{~J}_{\mathrm{H}-\mathrm{H} 1}=1.3 \mathrm{~Hz}, 1 \mathrm{H}, \mathrm{C}_{7} \mathrm{H}$ ), 7.89 (ddd, ${ }^{3} \mathrm{~J}_{\mathrm{H}-\mathrm{H} 1}=8.4,{ }^{3} \mathrm{~J}_{\mathrm{H}-\mathrm{H} 3}=7.0,{ }^{4} \mathrm{~J}_{\mathrm{H}-\mathrm{H} 2}=1.2 \mathrm{~Hz}, 1 \mathrm{H}, \mathrm{C}_{6} \mathrm{H}$ ), 7.47 $\left(\mathrm{t},{ }^{2} J_{\mathrm{H}-\mathrm{F}}=52.4 \mathrm{~Hz}, 1 \mathrm{H}, \mathrm{C}_{4} \mathrm{CHF}_{2}\right), 6.93\left(\mathrm{t},{ }^{2} \mathrm{~J}_{\mathrm{H}-\mathrm{F}}=53.6 \mathrm{~Hz}, 1 \mathrm{H}\right.$, $\mathrm{C}_{2} \mathrm{CHF}_{2}$ ) ppm. ${ }^{19} \mathrm{~F} \mathrm{NMR}\left(376 \mathrm{MHz}, \mathrm{CDCl}_{3}\right) \delta_{\mathrm{F}}=-109.07$ (dd, ${ }^{2} J_{F-H}$ $\left.=52.4,{ }^{5} J_{F-H}=2.0 \mathrm{~Hz}, \mathrm{C}_{4} \mathrm{CHF}_{2}\right),-113.13\left(\mathrm{~d},{ }^{2} J_{F-H}=53.7 \mathrm{~Hz}\right.$, $\left.\mathrm{C}_{2} \mathrm{CHF}_{2}\right)$ ppm. ${ }^{13} \mathrm{C} \mathrm{NMR}\left(101 \mathrm{MHz}, \mathrm{CDCl}_{3}\right) \delta_{\mathrm{C}}=150.64\left(\mathrm{t},{ }^{2} \mathrm{~J}_{\mathrm{C}-\mathrm{F}}=\right.$ $\left.26.9 \mathrm{~Hz}, \mathrm{C}_{2}\right), 148.16\left(\mathrm{~s}, \mathrm{C}_{9}\right), 144.60\left(\mathrm{t},{ }^{2} \mathrm{~J}_{\mathrm{C}-\mathrm{F}}=24.6 \mathrm{~Hz}, \mathrm{C}_{4}\right), 134.07$ $\left(\mathrm{s}, \mathrm{C}_{7}\right), 131.14$ and $\left(2{ }^{*} \mathrm{~s}, \mathrm{C}_{6}, 8\right), 125.70\left(\mathrm{t},{ }^{4} \mathrm{~J}_{\mathrm{C}-\mathrm{F}}=3.8 \mathrm{~Hz}, \mathrm{C}_{5}\right)$, 123.07 (s, $\left.\mathrm{C}_{10}\right), 114.09$ (t, ${ }^{1} \mathrm{~J}_{\mathrm{C}-\mathrm{F}}=243.4 \mathrm{~Hz}, \mathrm{C}_{2} \mathrm{CHF}_{2}$ ), 113.23 (t, $\left.{ }^{1} \mathrm{~J}_{\mathrm{C}-\mathrm{F}}=242.7 \mathrm{~Hz}, \mathrm{C}_{4} \mathrm{CHF}_{2}\right), 112.20\left(\mathrm{~s}, \mathrm{C}_{3} \mathrm{CN}\right), 102.62\left(\mathrm{t},{ }^{3} \mathrm{~J}_{\mathrm{C}-\mathrm{F}}=6.6\right.$ $\left.\mathrm{Hz}, \mathrm{C}_{3}\right)$ ppm. IR $\vee\left(\mathrm{cm}^{-1}\right): 2988-2901\left(\mathrm{C}_{\mathrm{sp} 3} \mathrm{H}\right), 2234(\mathrm{CN})$. $\mathrm{C}_{12} \mathrm{H}_{6} \mathrm{~F}_{4} \mathrm{~N}_{2}$ (254): calcd (\%) N 11.02, C $56.70, \mathrm{H} 2.38$, found $\mathrm{N}$ 10.96, C 56.28, H 2.43. MP: $130.6-132.3^{\circ} \mathrm{C}$.

\section{2,4-Bis(difluoromethyl)-3-(tetramethyl-1,3,2-dioxaborolan-2- yl)quinoline $12 \mathrm{aA}$}

To a solution of 2,4-bis(difluoromethyl)-3-iodoquinoline 9aA (1 equiv., $2.42 \mathrm{~g}, 6.82 \mathrm{mmol}$ ) and 2-isopropoxy-4,4,5,5-tetramethyl1,3,2-dioxaborolane (3 equiv., $4.17 \mathrm{~mL}, 20.4 \mathrm{mmol}$ ) in anhydrous THF $(10 \mathrm{ml})$ at $-10{ }^{\circ} \mathrm{C}$ was added dropwise $\mathrm{PrMgCl}$.LiCl $(1.5$ equiv., $1.3 \mathrm{M}$ in THF, $7.86 \mathrm{~mL}, 10.2 \mathrm{mmol}$ ). The reaction mixture was allowed to warm up to r.t. overnight. It was quenched with a saturated aqueous solution of ammonium chloride $\left(\mathrm{NH}_{4} \mathrm{Cl}\right)$ and the aqueous phase was extracted with $\mathrm{Et}_{2} \mathrm{O}$. The combined organic layers were washed with saturated aqueous $\mathrm{NH}_{4} \mathrm{Cl}$ then brine, dried over $\mathrm{Na}_{2} \mathrm{SO}_{4}$ and concentrated under reduced pressure. 2,4-Bis(difluoromethyl)-3-(tetramethyl-1,3,2dioxaborolan-2-yl)quinoline 12aA was obtained after purification on column chromatography using a gradient of AcOEt in cyclohexane $(0-5 \%)$ as a colourless solid in $54 \%$ yield $(1.31 \mathrm{~g}) .{ }^{1} \mathrm{H}$ NMR $\left(400 \mathrm{MHz}, \mathrm{CDCl}_{3}\right) \delta_{\mathrm{H}}=8.30\left(\mathrm{~d},{ }^{3} \mathrm{H}_{\mathrm{H}-\mathrm{H}}=8.5 \mathrm{~Hz}, 1 \mathrm{H}, \mathrm{C}_{5} \mathrm{H}\right)$, 
$8.21\left(\mathrm{~d},{ }^{3} \mathrm{~J}_{\mathrm{H}-\mathrm{H}}=8.4 \mathrm{~Hz}, 1 \mathrm{H}, \mathrm{C}_{8} \mathrm{H}\right), 7.86-7.80\left(\mathrm{~m}, 1 \mathrm{H}, \mathrm{C}_{7} \mathrm{H}\right), 7.71$ $\left(\mathrm{t},{ }^{3} \mathrm{~J}_{\mathrm{H}-\mathrm{H}}=7.2 \mathrm{~Hz}, 1 \mathrm{H}, \mathrm{C}_{6} \mathrm{H}\right), 7.34\left(\mathrm{t},{ }^{2} \mathrm{~J}_{\mathrm{H}-\mathrm{F}}=53.9 \mathrm{~Hz}, 1 \mathrm{H}, \mathrm{C}_{4} \mathrm{CHF}_{2}\right)$, $6.98\left(\mathrm{t},{ }^{2} \mathrm{~J}_{\mathrm{H}-\mathrm{F}}=55.2 \mathrm{~Hz}, 1 \mathrm{H}, \mathrm{C}_{2} \mathrm{CHF}_{2}\right), 1.45(\mathrm{~s}, 12 \mathrm{H}, \mathrm{Bpin}) \mathrm{ppm} .{ }^{19} \mathrm{~F}$ $\operatorname{NMR}\left(376 \mathrm{MHz}, \mathrm{CDCl}_{3}\right) \delta_{\mathrm{F}}=-108.94\left(\mathrm{~d},{ }^{2} J_{F-H}=53.8 \mathrm{~Hz}, \mathrm{C}_{4} \mathrm{CHF}_{2}\right)$, $-112.64\left(\mathrm{~d},{ }^{2} J_{F-H}=55.2 \mathrm{~Hz}, \mathrm{C}_{2} \mathrm{CHF}_{2}\right) \mathrm{ppm} .{ }^{11} \mathrm{~B} \mathrm{NMR}(128 \mathrm{MHz}$, $\left.\mathrm{CDCl}_{3}\right) \delta_{\mathrm{B}}=31.01$ (s, Bpin) ppm. ${ }^{13} \mathrm{C} \mathrm{NMR}\left(101 \mathrm{MHz}, \mathrm{CDCl}_{3}\right) \delta_{\mathrm{C}}$ $=154.17\left(\mathrm{t},{ }^{2} J_{C-F}=24.8 \mathrm{~Hz}, \mathrm{C}_{2}\right), 147.70\left(\mathrm{~s}, \mathrm{C}_{9}\right), 144.29\left(\mathrm{t},{ }^{2} J_{\mathrm{C}-F}=\right.$ $\left.21.9 \mathrm{~Hz}, \mathrm{C}_{4}\right), 131.15\left(\mathrm{~s}, \mathrm{C}_{7}\right), 130.67$ (s, $\left.\mathrm{C}_{8}\right), 129.12\left(\mathrm{~s}, \mathrm{C}_{6}\right), 124.57$ $\left(\mathrm{t},{ }^{4} \mathrm{~J}_{\mathrm{C}-\mathrm{F}}=2.9 \mathrm{~Hz}, \mathrm{C}_{5}\right), 124.07\left(\mathrm{~s}, \mathrm{C}_{10}\right), 114.83\left(\mathrm{t},{ }^{1} \mathrm{~J}_{\mathrm{C}-\mathrm{F}}=242.4 \mathrm{~Hz}\right.$, $\mathrm{C}_{4} \mathrm{CHF}_{2}$ ), $114.57\left(\mathrm{t},{ }^{1} \mathrm{~J}_{\mathrm{C}-\mathrm{F}}=241.4 \mathrm{~Hz}, \mathrm{C}_{2} \mathrm{CHF}_{2}\right), 85.62\left(\mathrm{~s}, \mathrm{C}_{11}\right)$, $25.19\left(\mathrm{~s}, \mathrm{C}_{11} \mathrm{CH}_{3}\right)$ ppm. $\mathrm{C}_{17} \mathrm{H}_{18} \mathrm{~F}_{4} \mathrm{NBO}_{2}$ (355): calcd (\%) N 3.94, C 57.49, H 5.11, found N 4.01, C 57.68, H 5.20. MP: 82.6-83.5 ${ }^{\circ} \mathrm{C}$.

\section{Acknowledgements}

We thank the CNRS France (Centre National de la Recherche Scientifique), the University of Strasbourg, and we are very much grateful to Bayer S.A.S. for a grant to F. Aribi. The French Fluorine Network (GIS Fluor) is also acknowledged.

Keywords: Fluorine $\cdot$ Quinoline $\cdot$ Heterocycles $•$ Cyclization • FARs

[1] a) P. Kirsch, Modern Fluoroorganic Chemistry: Synthesis, Reactivity, Applications, Wiley- $\mathrm{VCH}$, Weinheim, Germany, 2004; b) J. Wang, M. Sánchez-Roselló, J. L. Aceña, C. del Pozo, A. E. Sorochinsky, S. Fustero, V. A. Soloshonok, H. Liu, Chem. Rev. 2014, 114, 2432-2506; c) W. K. Hagmann, J. Med. Chem. 2008, 51, 4359-4369; d) K. Müller, C. Faeh, F. Diederich, Science 2007, 317, 1881-1886; e) J.-P. Bégué, D. BonnetDelpon, Bioorganic and Medicinal Chemistry of Fluorine, John Wiley \& Sons, Hoboken, New Jersey, 2008; f) Y. Zhou, J. Wang, Z. Gu, S. Wang, W. Zhu, J. L. Aceña, V. A. Soloshonok, K. Izawa, H. Liu, Chem. Rev. 2016, 116, 422-518; g) S. Purser, P. R. Moore, S. Swallow, V. Gouverneur, Chem. Soc. Rev. 2008, 37, 320-330; h) K. M. Veronique Gouverneur, Fluorine in Pharmaceutical and Medicinal Chemistry, Imperial College Press, London, 2012; i) V. Gouverneur, K. Seppelt, Chem. Rev. 2015, 115, 563-565; j) G. Theodoridis, in Advances in Fluorine Science, Vol. 2 (Ed.: A. Tressaud), Elsevier B.V, 2006, pp. 120175 ; k) J. Liu, A. Maisonial-Besset, B. Wenzel, D. Canitrot, A. Baufond, J.-M. Chezal, P. Brust, E. Moreau, Eur. J. Med. Chem. 2017, 136, 548560.

[2] a) M. Schlosser, Angew. Chem. Int. Ed. 2006, 45, 5432-5446; b) J.-A Ma, D. Cahard, Chem. Rev. 2008, 108, PR1-PR43; c) O. A. Tomashenko, V. V. Grushin, Chem. Rev. 2011, 111, 4475-4521; d) P. B. Arockiam, L. Guillemard, J. Wencel - Delord, Adv. Synth. Catal. 2017, 359, 25712579; e) X. Geng, F. Lin, X. Wang, N. Jiao, J. Photochem. Photobiol. A: Chem. 2017; f) T. T. Tung, S. B. Christensen, J. Nielsen, Chem. Eur. J. 2017, 23, 18125-18128.

[3] a) D. A. Kummer, M. D. Cummings, M. Abad, J. Barbay, G. Castro, R. Wolin, K. D. Kreutter, U. Maharoof, C. Milligan, R. Nishimura, J. Pierce, C. Schalk-Hihi, J. Spurlino, M. Urbanski, H. Venkatesan, A. Wang, C.
Woods, X. Xue, J. P. Edwards, A. M. Fourie, K. Leonard, Bioorg. Med. Chem. Lett. 2017, 27, 2047-2057; b) D. M. Ferguson, J. R. Bour, A. J Canty, J. W. Kampf, M. S. Sanford, J. Am. Chem. Soc. 2017, 139, 1166211665 ; c) J. C. Sloop, C. L. Bumgardner, W. D. Loehle, J. Fluorine Chem. 2002, 118, 135-147; d) J. C. Sloop, J. Phys. Org. Chem. 2009, 22, 110117; e) Y. Fujiwara, J. A. Dixon, R. A. Rodriguez, R. D. Baxter, D. D. Dixon, M. R. Collins, D. G. Blackmond, P. S. Baran, J. Am. Chem. Soc 2012, 134, 1494-1497.

[4] R. D. Taylor, M. MacCoss, A. D. G. Lawson, J. Med. Chem. 2014, 57, 5845-5859.

[5] a) A. V. Fokin, A. F. Kolomiyets, J. Fluorine Chem. 1988, 40, 247-259; b) X. G. Hu, L. Hunter, Beilstein J Org Chem 2013, 9, 2696-2708; c) V. A. Petrov, in Fluorinated Heterocyclic Compounds: Synthesis, Chemistry, and Applications, Wiley, 2009, p. 533.

[6] F. Aribi, E. Schmitt, A. Panossian, J.-P. Vors, S. Pazenok, F. R. Leroux, Org. Chem. Front. 2016, 3, 1392-1415.

[7] B. Duda, S. N. Tverdomed, B. S. Bassil, G.-V. Röschenthaler, Tetrahedron 2014, 70, 8084-8096.

[8] a) E. Schmitt, B. Commare, A. Panossian, J.-P. Vors, S. Pazenok, F. R. Leroux, Chem. Eur. J., DOI: 10.1002/chem.201703982; b) E. Schmitt, G. Landelle, J.-P. Vors, N. Lui, S. Pazenok, F. R. Leroux, Eur. J. Org. Chem. 2015, 6052-6060; c) E. Schmitt, B. Rugeri, A. Panossian, J.-P. Vors, S. Pazenok, F. R. Leroux, Org. Lett. 2015, 17, 4510-4513; d) E. Schmitt, A. Panossian, J.-P. Vors, C. Funke, N. Lui, S. Pazenok, F. R. Leroux, Chem. Eur. J. 2016, 22, 11239-11244; e) B. Commare, E. Schmitt, F. Aribi, A. Panossian, J.-P. Vors, S. Pazenok, F. R. Leroux, Molecules 2017, 22, 977-1003; f) E. Schmitt, S. Bouvet, B. Pégot, A. Panossian, J.-P. Vors, S. Pazenok, E. Magnier, F. R. Leroux, Org. Lett. 2017, 19, 4960-4963.

[9] a) V. A. Petrov, S. Swearingen, W. Hong, W. Chris Petersen, J. Fluorine Chem. 2001, 109, 25-31; b) V. A. Petrov, Adv. Org. Synth. 2006, 2, 269290; c) L. M. Grieco, G. A. Halliday, C. P. Junk, S. R. Lustig, W. J. Marshall, V. A. Petrov, J. Fluorine Chem. 2011, 132, 1198-1206.

[10] a) N. N. Yarovenko, M. A. Raksha, Zh. Obshch. Khim. 1959, 29, 21592163; b) N. N. Yarovenko, M. A. Raksha, J. Gen. Chem. USSR (Engl. Transl.) 1959, 29, 2125-2128.

[11] A. Takaoka, H. Iwakiri, N. Ishikawa, Bull. Chem. Soc. Jpn. 1979, 52, 3377-3380.

[12] S. Perrone, F. Rosato, A. Salomone, L. Troisi, Tetrahedron 2013, 69, 3878-3884.

[13] A. Sagar, S. Vidaycharan, A. H. Shinde, D. S. Sharada, Org. Biomol. Chem. 2016, 14, 4018-4022.

[14] W.-H. Miller, M. B. Rouse, M.-A. Seefeld, 2006, WO 2006/081289.

[15] M. H. T. Bui, Y. Chen, T. D. Cushing, J. A. Duquette, B. Fisher, F. Gonzalez Lopez De Turiso, X. Hao, X. He, M. G. Johnson, B. Lucas, 2010, WO 2010/151740.

[16] C. Boezio, H. Bregman, J. R. Coats, E. F. Dimauro, T. Dineen, B. Du, R. Graceffa, C. Kreiman, D. La, I. E. Marx, N. Chakka, H. N. Nguyen, E. A. Peterson, M. Weiss, K. W. Copeland, H. L. Deak, A. Boezio, 2013, WO 2013/086229.

[17] E. Demory, V. Blandin, J. Einhorn, P. Y. Chavant, Org. Proc. Res. Dev. 2011, 15, 710-716.

[18] I. G. Maslennikov, K. I. Eremin, Russ. J. Gen. Chem. 2011, 81, 1741 